\title{
The Principle of Limiting Absorption for Second-order Differential Equations with Operator-valued Coefficients*
}

\author{
By \\ Yoshimi SAITō**
}

\section{§0. Introduction}

Let us consider differential operators of the form

$$
L=-\frac{d^{2}}{d r^{2}}+B(r)+C(r) \quad(0<r<\infty),
$$

where for each $r \in(0, \infty) B(r)$ and $C(r)$ are operators in a Hilbert space $X$. $\quad L$ acts on $X$-valued funcitons on $(0, \infty)$.

The purpose of the present paper is to justify the principle of limiting absorption for the equation

$$
(L-(\lambda+i \mu)) u=f .
$$

The essence of the above principle consists in the following: Let $u_{\lambda+i \mu}$ be the solution of $(0.2)$, where $f$ is a given $X$-valued function on $(0, \infty)$. Then a solution $u_{\lambda}$ of the equation

$$
(L-\lambda) u=f
$$

is given by $u_{\lambda}=\lim _{\mu \rightarrow 0} u_{\lambda+i \mu}$. The meaning of the limit is to be determined suitably. For the literature of the principle of limiting absorption see, for example, Eidus [1].

Jäger [5] considers the differential operator $L$ and gives, among others, the following result: Let $B(r)$ be a non-negative self-adjoint

Received July 2, 1971.

* Thesis presented to the Kyoto University.

** Department of Mathematics, Osaka City University, Sugimoto-cho, Sumiyoshi-ku, Osaka, 558, Japan. 
operator in $X$ and let $C(r)$ behave like $0\left(r^{-\frac{3}{2}-\varepsilon}\right)(\varepsilon>0)$ at infinity. Then with some other conditions imposed on $B(r)$ and $C(r)$ the principle of limiting absorption holds for equation (0.2) with boundary condition

$$
u(0)=0
$$

and the "radiation condition"

$$
\int_{0}^{\infty}\left|u^{\prime}(r)-i \sqrt{z} u(r)\right|^{2} d r<\infty \quad(z=\lambda+i \mu)
$$

where | | means the norm of $X$. He uses the above results to construct an eigenfunction expansion associated with $L$.

We shall extend Jäger's results to $L$ with $C(r)$ which behaves like $0\left(r^{-1-\varepsilon}\right)(\varepsilon>0)$ at infinity. In our case the radiation condition (0.5) will be replaced by

$$
\int_{0}^{\infty}(1+r)^{-1+\varepsilon}\left|u^{\prime}(r)-i \sqrt{z} u(r)\right|^{2} d r<\infty
$$

which is weaker than (0.5).

As an application we shall prove the principle of limiting absorption for the Schrödinger operator $-\Delta+q(y)$ in $\mathbf{R}^{n}(n \geqq 3)$ with $q(y)$ $=0\left(|y|^{-1-\varepsilon}\right)$ at infinity. In this case $X=L^{2}\left(S^{n-1}\right)$ and

$$
\left\{\begin{array}{l}
B(r)=\frac{1}{r^{2}}\left\{-\Lambda_{n}+\frac{(n-3)(n-1)}{4}\right\} \\
C(r)=q(r \omega) \times \quad\left(r=|y|, \omega=\frac{y}{r} \in S^{n-1}\right),
\end{array}\right.
$$

where $S^{n-1}$ is $(n-1)$-sphere, and $\Lambda_{n}$ is the Laplace-Beltrami operator on $S^{n-1}$.

In $\S 1$ we state conditions imposed on $B(r)$ and $C(r)$ and prove some inequalities which will be used to obtain various a priori estimates for the solution of equation (0.2) in $\S 3 . \quad \S 2$ and $\S 3$ are devoted to showing the existence and uniqueness of the solution $u$ of the equation

$$
\left(L-k^{2}\right) u=f \quad(\operatorname{Im} k \geqq 0)
$$


which satisfies the boundary condition (0.4) and the radiation condition (0.6). Moreover we show that the solution $u$ continuously depends on $k$. Thus the principle of limiting absorption is justified. We discuss in $\S 4$ the dependency on $C(r)$ of the solution of equation (0.8). In $\S 5$ we apply these results to the Schrödinger operator in $\mathbf{R}^{n}(n \geqq 3)$.

Using the results obtained in this paper we can develop a spectral and scattering theory for the differential operator $L$ with an application to Schrödinger operators $-\Delta+q(y)$ in $\mathbf{R}^{n}$, where $q(y)=0\left(|y|^{-1-\varepsilon}\right)$ at infinity. We shall discuss these elsewhere. ${ }^{1)}$

Recently we have been informed by Prof. T. Ikebe that the following very extensive results have been obtained by S. Agmon: Let

$$
L=\sum_{|\alpha| \leqq m} a_{\alpha} D^{\alpha}=L_{0}+B
$$

be an elliptic operator in $\mathbf{R}^{n}$ which has a unique self-adjoint extension in $L^{2}\left(\mathbf{R}^{n}\right)$, where $L_{0}=\sum_{|\alpha| \leqq m} a_{\alpha}^{0} D^{\alpha}$ is an elliptic operator with constant coefficients, and $B=\sum_{|\alpha| \leqq m} b_{\alpha} D^{\alpha}$ is a differential operator with $b(x)=0\left(|x|^{-1-\varepsilon}\right)$ as $|x|$ $\rightarrow \infty$. Assume that $\lambda>0$ does not belong to an exceptional set which is discrete in $(-\infty, \infty)$ and contains all the eigenvalues of $L$. Then the principle of limiting absorption holds good for $\lambda$, i.e., we have

$$
\begin{aligned}
& v_{\lambda \pm i \mu} \rightarrow v_{\lambda \pm i 0} \text { as } \mu \downarrow 0 \text { in } L_{2}\left(\mathbf{R}^{n},(1+|x|)^{-1-\varepsilon} d x\right), \\
& \left.\left|\int_{R^{n}}(1+|x|)^{-1-\varepsilon}\right| v_{\lambda \pm i \mu}(x)\right|^{2} d x \leqq C \int_{R^{n}}(1+|x|)^{1+\varepsilon}|f(x)|^{2} d x,
\end{aligned}
$$

where $v_{\lambda \pm i \mu}=(L-(\lambda \pm i \mu))^{-1} f$. In his method any radiation condition is unnecessary. These results are used to construct an eigenfunction expansion for $L$.

\section{§1. Assumptions and Preliminary Lemmas}

Let $X$ be a Hilbert space with the norm | | and inner product $($,$) .$ For an open interval $J$ in $\mathbf{R}^{2)}$ and $\beta \in \mathbf{R}$ we denote by $H^{\beta}(J, X)$ the

1) See Y. Saitō [7].

2) $\mathbf{R}$ is the set of all real numbers. 
Hilbert space of all (equivalence classes of) $X$-valued function on $J$ with the norm and inner product

$$
\left\{\begin{array}{l}
\|f\|_{\beta, J}=\left[((f, f))_{\beta, J}\right]^{\frac{1}{2}}, \\
((f, g))_{\beta, J}=\int_{J}(f(r), g(r))(1+|r|)^{\beta} d r .
\end{array}\right.
$$

Let $Y$ be a linear topological space, let $m$ be a non-negative integer, and let $J=\left(a_{1}, a_{2}\right)$ be an open interval in $\mathbf{R}$. $C^{m}(J, Y)$ denotes the set of all $Y$-valued functions on $J$ having $m$ strong continuous derivatives. We denote by $C^{m}(\bar{J}, Y)^{3)}$ the set of all $Y$-valued functions $f(r)$ such that $f \in C^{m}(J, Y)$ and $\frac{d^{j} f}{d r^{j}} \quad(j=0,1, \ldots, m)$ can be extended to continuous functions on $\bar{J} . C_{0, a_{i}}^{m}(J, Y)(i=1,2)$ denotes the set of all $f \in C^{m}(J, Y)$ satisfying $f(r)=0$ in some neighborhood of $a_{i}$. We put $C_{0}^{m}(J, Y)$ $=C_{0, a_{1}}^{m}(J, Y) \cap C_{0, a_{2}}^{m}(J, Y)$. If $Y=\mathbf{C},{ }^{4)}$ we omit $\mathbf{C}$ as in $C^{m}(J)=C^{m}(J, \mathbf{C})$.

Let $I=(0, \infty)$ and let $B(r)$ and $C(r)$ be operator-valued functions on I. For local properties of $B(r)$ and $C(r)$ we make the following

Assumption 1.1. (a) For each $r \in I B(r)$ is a non-negative, selfadjoint operator in $X$ such that its domain $\mathscr{D}(B(r))=D^{5)}$ does not depend on $r$, and $B(r) x \in C^{0}(I, X)$ for any $x \in D$.

(b) Let $x, y \in D$. Then $(B(r) x, y) \in C^{2}(I)$ and for any compact interval $M \subset I$ there exists a constant $c_{1}(M)>0$ satisfying

$$
\left|\frac{d^{j}}{d s^{j}}(B(s) x, y)\right| \leqq c_{1}(M)\left(|x|+\mid B^{\frac{1}{2}}(r) x\right)\left(|y|+\left|B^{2}(r) y\right|\right)
$$

where $r, s \in M$ and $j=1,2$.

(c) For each $r \in I \quad C(r)$ is a symmetric operator in $X$ with $\mathscr{D}(C(r))$ $=D$ such that $C(r) x \in C^{1}(I, X)$ for any $x \in D$.

(d) Let $M$ be a compact interval in $I$. Then there exists a constant $c_{2}(M)>0$ such that

3) $\bar{J}$ means the closure of $J$.

4) $\mathbf{C}$ is all complex numbers.

5) $\mathscr{D}(T)$ means the domain of $T$. 


$$
\left|\frac{d}{d r} C(r) x\right| \leqq c_{2}(M)\left(|x|+\left|B^{2}(r) x\right|\right)
$$

holds for any $x \in D$ and any $r \in M$.

We introduce the norm \|\|$_{B, J}$ and inner product $((,))_{B, J}$ by

$$
\begin{gathered}
\|f\|_{B, J}=\left[((f, f))_{B, J}\right]^{\frac{1}{2}}, \\
\left.((f, g))_{B, J}=\left(\left(f^{\prime}, g^{\prime}\right)\right)_{0, J}+((B f, g))_{0, J}+((f, g))_{0, J \cdot}{ }^{6}\right)
\end{gathered}
$$

We denote by $C^{2, B}(J, X)\left(C_{0, a_{i}}^{2, B}(J, X), i=1,2\right)$ the linear space spanned by the set of all $\varphi \in C^{2}(J, X)$ having the form $\varphi=\psi x$, where $x \in D$, $\psi \in C^{2}(J)\left(\psi \in C_{0, a_{i}}^{2}(J), i=1,2\right)$ and $\|\varphi\|_{B, J}<\infty$. We denote $C_{0, a_{1}}^{2, B}(J, X)$ $\cap C_{0, a_{2}}^{2, B}(J, X)$ by $C_{0}^{2, B}(J, X)$. We define Hilbert spaces $H^{1, B}(J, X)$, $H_{0}^{1, B}(J, X)$ and $H_{0, a_{i}}^{1, B}(J, X)(i=1,2)$, respectively, by the completion of $C^{2, B}(J, X), C_{0}^{2, B}(J, X)$ and $C_{0, a_{i}}^{2, B}(J, X)(i=1,2)$ in the norm \|\|$_{B, J}$. Let us denote by $\operatorname{loc} H^{0}(\bar{I}, X)$ the set of all $X$-valued functions $f(r)$ on $I$ such that $f \in H^{0}((0, b), X)$ for any $b>0$. In a similar way $\operatorname{loc} H^{1, B}(\bar{I}, X)$ and $\operatorname{loc} H_{0}^{1, B}(\bar{I}, X)$ are also defined.

Assumption 1.2. ${ }^{7)}$ (a) There exist constants $\rho_{1}>0$ and $c_{1}>1$ such that

$$
-\frac{d}{d r}(B(r) x, x) \geqq \frac{c_{1}}{r}(B(r) x, x)
$$

holds for any $x \in D$ and any $r \geqq \rho_{1}$.

(b) For each finite $b \in I$ the natural imbedding

$$
H_{0}^{1} B((0, b), X) \rightarrow H^{0}((0, b), X)
$$

is compact.

(c) There exists $c_{2}>0$ such that

6) Here and in the sequel $u^{\prime}$ and $u^{\prime \prime}$ mean $\frac{d u}{d r}$ and $\frac{d^{2} u}{d r^{2}}$, respectively.

7) The conditions imposed on $B(r)$ and $C(r)$ are the same as in Jäger [5] except (c) of Assumption 1.2. Jäger [5] assumes that

$$
|C(r) x| \leqq c_{2}(1+r)^{-\frac{3}{2}-\varepsilon}\left(|x|+\left|B^{\frac{1}{2}}(r) x\right|\right), \quad(r \in I, x \in D)
$$

instead of (1.8). 


$$
|C(r) x| \leqq c_{2}(1+r)^{-1-\varepsilon}\left(|x|+\left|B^{\frac{1}{2}}(r) x\right|\right), \quad(r \in I, x \in D)
$$

with some $0<\varepsilon<1$.

For an open interval $J \subset I \mathscr{U}(J)$ denotes the set of all linear, continuous functionals on $H_{0}^{1, B}(J, X) . \mathscr{U}(J)$ is a Banach space with the norm

$$
\|l\|_{J}=\sup \left\{\mid\langle l, \varphi>| ; \varphi \in C_{0}^{2, B}(J, X),\|\varphi\|_{B}=1\right\} .
$$

For example, for $g \in H^{0}(J, X)$ we define $l[g] \in \mathscr{U}(J)$ by

$$
\left\langle l[g], \varphi>=((g, \varphi))_{0, J} \quad\left(\varphi \in H_{0}^{1, B}(J, X)\right) .\right.
$$

Then we can easily see

$$
\|l[g]\|_{J} \leqq\|g\|_{0, J}
$$

Definition 1.3. Let $l \in \mathscr{U}(I), u \in H^{1, B}(I, X)$ and $k \in \mathbb{C}^{+}$be given, where

$$
\mathbf{C}^{+}=\{k \mid k \in \mathbf{C}, \operatorname{Im} k \geqslant 0 \text { and } \operatorname{Re} k \neq 0\} .{ }^{8)}
$$

Then $v \in \operatorname{loc} H^{1, B}(\bar{I}, X)$ is called a radiative function for $\{L, k, l, u\}$, if the following three conditions hold:

(a) $v-u \in \operatorname{loc} H_{0}^{1, B}(\bar{I}, X)$.

(b) $v^{\prime}-i k v \in H^{-1+\varepsilon}(I, X)$ (the "radiation condition") $)^{9)}$

(c) For all $\varphi \in C_{0}^{2, B}(I, X)$ we have

$$
\left(\left(v,\left(L-\bar{k}^{2}\right) \dot{\varphi}\right)\right)_{0, I}=\langle l, \varphi>\text {. }
$$

We shall give a lemma which will be used to prove the existence theorem of the radiative fucntion.

Lemma 1.4. ${ }^{10)}$ Let $I_{0}=(b, \infty), b>0$. Eor each $r \in I_{0} \quad B(r)$ is assumed

8) $\operatorname{Im} k$ and $\operatorname{Re} k$ mean the imaginary and real, respectively.

9) In Jäger [5] the radiation condition is defined by $v^{\prime}-i k v \in H^{0}(I, X)$.

10) Cf. Jäger [3], Hilfssatz 4 (p. 68). 
to be a non-negative, self-adjoint operator in $X$ with $\mathscr{D}(B(r))=D$ constant in r. Suppose that $(B(r) x, x) \in C^{1}\left(I_{0}\right)$ for any $x \in D$ and that we have

$$
-\frac{d}{d r}(B(r) x, x) \geqq \frac{e_{0}}{r}(B(r) x, x) \quad\left(x \in D, r \geqq b_{0}\right)
$$

with constants $b_{0}>b$ and $e_{0}>1$. Let $C(r), r \in I_{0}$, be a symmetric operator with $\mathscr{D}(C(r))=D$. Let $v(r)$ be an $X$-valued function on $I_{0}$ which satisfies the following (i) (iii):

(i) $v \in C^{2}\left(I_{0}, D\right),{ }^{11)} \quad B v, C v \in C^{0}\left(I_{0}, X\right)$, and

$$
\left(-\frac{d^{2}}{d r^{2}}+B(r)+C(r)-k^{2}\right) v(r)=g(r) \quad\left(r \in I_{0}\right)
$$

with $k \in \mathbf{C}^{+}$and $g \in H^{1+\varepsilon}\left(I_{0}, X\right)$.

(ii) $v^{\prime}-i k v \in H^{-1+\varepsilon}\left(I_{0}, X\right)$ and $v \in H^{-1-\varepsilon}\left(I_{0}, X\right)$.

(iii) We have

$$
|C(r) v(r)|^{2} \leqq e_{1} r^{-2-2 \varepsilon}\left(\left|v^{\prime}(r)\right|^{2}+\left|B^{\frac{1}{2}}(r) v(r)\right|^{2}\right) \quad(r \geqq b)
$$

with constants $e_{1}>0,0<\varepsilon<1$.

Then there exist constants $\delta_{0}>0$ and $r_{0} \geqq b_{0}+1$ which do not depend on $v(r)$ and $g(r)$ such that

$$
\begin{gathered}
\int_{r_{0}+1}^{\infty} r^{-1+\varepsilon}\left\{\left|v^{\prime}(r)-i k v(r)\right|^{2}+(B(r) v(r), v(r))\right\} d r \\
\leqq \delta_{0} \int_{r_{0}-1}^{\infty}\left(r^{-1-\varepsilon}|v(r)|^{2}+r^{1+\varepsilon}|g(r)|^{2}\right) d r
\end{gathered}
$$

Moreover $\delta_{0}$ and $r_{0}$, as functions of $k$, are bounded on any bounded set in $\mathbf{C}^{+}$.

For the proof of this lemma we need the following lemma due to Jäger [5] (Lemma 4.1).

Lemma 1.5. Let $-\infty \leqq a_{2}<a_{1}<b_{1}<b_{2} \leqq \infty$ and put $I_{i}=\left(a_{i}, b_{i}\right)$, 11) $C^{2}\left(I_{0}, D\right)$ is the set of all $\varphi(r) \in C^{2}\left(I_{0}, X\right)$ such that $\varphi(r) \in D$ for any $r \in I_{0}$. 
$i=1,2$. Let $B(r)$ be a non-negative, self-adjoint operator in $X$ for each $r \in I_{2}$ with $\mathscr{D}(B(r))=D$ constant in $r$. Let $C(r)$ be a symmetric operator in $X$ with $\mathscr{D}(C(r))=D$ for each $r \in I_{2}$. Suppose that $v \in H^{1, B}\left(I_{2}, X\right)$ satisfies for any $\varphi \in C_{0}^{2, B}\left(I_{2}, X\right)$

$$
\left(\left(v,\left(-\frac{d^{2}}{d r^{2}}+B(\cdot)+C(\cdot)-k^{2}\right) \varphi\right)\right)_{0, I_{2}}=\langle l, \varphi\rangle
$$

where $k \in \mathbf{C}^{+}$and $l \in \mathscr{U}\left(I_{2}\right)$. Suppose, further, that $v$ satisfies

$$
|C(r) v(r)| \leqq c_{2}\left(\left|v^{\prime}(r)\right|+\left|B^{\frac{1}{2}}(r) v(r)\right|+|v(r)|\right), \quad\left(r \in I_{2}\right)
$$

with a constant $c_{2}=c_{2}\left(I_{2}\right)>0$. Then there exists a constant $K=K\left(I_{1}, I_{2}, k\right)$ $>0$ such that

$$
\|v\|_{B, I_{1}} \leqq K\left(\|v\|_{0, I_{2}}+\|l\|_{I_{2}}\right)
$$

holds. Further if we assume $v \in H_{0, a_{2}}^{1, B}\left(I_{2}, X\right)$ and $a_{2}>-\infty$ then the conclusion is valid for $a_{2} \leqq a_{1}$.

Proof of Lemma 1.4. Take $r_{0} \geqq b_{0}+1$, where $b_{0}$ is given in (1.14). Let $\psi \in C^{1}\left(I_{0}\right)$ such that $0 \leqq \psi \leqq 1, \psi^{\prime}(r) \geqq 0$, and

$$
\varphi(r)= \begin{cases}0 & \text { for } r \in\left(b, r_{0}\right], \\ 1 & \text { for } r \in\left[r_{0}+1, \infty\right) .\end{cases}
$$

Then we have for $r \geqq r_{0}$

$$
\begin{aligned}
& \frac{d}{d r}\left(r^{\varepsilon} \psi(r)\left|v^{\prime}(r)-i k v(r)\right|^{2}\right) \\
& =\varepsilon r^{-1+\varepsilon} \psi(r)\left|v^{\prime}(r)-i k v(r)\right|^{2}+r^{\varepsilon} \psi^{\prime}(r)\left|v^{\prime}(r)-i k v(r)\right|^{2} \\
& \quad+2 r^{\varepsilon} \psi(r) \operatorname{Re}\left(v^{\prime \prime}(r)-i k v^{\prime}(r), v^{\prime}(r)-i k v(r)\right) \\
& \geqq \\
& \quad \varepsilon r^{-1+\varepsilon} \psi(r)\left|v^{\prime}-i k v\right|^{2}+2 r^{\varepsilon} \psi(r) \operatorname{Re}\left(v^{\prime \prime}-i k v^{\prime}, v^{\prime}-i k v\right),
\end{aligned}
$$

since we have assumed that $\psi^{\prime}(r) \geqq 0$. Noting that $\operatorname{Im} k \geqq 0$ we have

$$
\operatorname{Re}\left(v^{\prime \prime}(r)-i k v^{\prime}(r), v^{\prime}(r)-i k v(r)\right)
$$




$$
\begin{aligned}
= & \operatorname{Re}\left(v^{\prime \prime}(r)-B(r) v(r)+k^{2} v(r), v^{\prime}(r)-i k v(r)\right) \\
& +(\operatorname{Im} k)\left\{\left|v^{\prime}(r)-i k v(r)\right|^{2}+(B(r) v(r), v(r))\right\} \\
& +\operatorname{Re}\left(B(r) v(r), v^{\prime}(r)\right) \\
\geqq & \operatorname{Re}\left(v^{\prime \prime}(r)-B(r) v(r)+k^{2} v(r), v^{\prime}(r)-i k v(r)\right) \\
& +\operatorname{Re}\left(B(r) v(r), v^{\prime}(r)\right) .
\end{aligned}
$$

We estimate $2 r^{\varepsilon} \operatorname{Re}\left(v^{\prime \prime}-B v+k^{2} v, v^{\prime}-i k v\right)$ as follows:

$$
\begin{aligned}
& 2 r^{\varepsilon} \operatorname{Re}\left(v^{\prime \prime}(r)-B(r) v(r)+k^{2} v(r), v^{\prime}(r)-i k v(r)\right) \\
& \geqq-2 r^{\varepsilon}\left|v^{\prime \prime}(r)-B(r) v(r)+k^{2} v(r)\right|\left|v^{\prime}(r)-i k v(r)\right| \\
& \geqq-\frac{r^{2 \beta}}{\alpha}\left|v^{\prime \prime}(r)-B(r) v(r)+k^{2} v(r)\right|^{2}-\alpha r^{2 \eta}\left|v^{\prime}(r)-i k v(r)\right|^{2} \\
& \qquad(\alpha>0, \beta+\eta=\varepsilon) \\
& \geqq-\frac{\delta_{1}}{\alpha}\left[r^{-2-2 \varepsilon+2 \beta}\left\{|v(r)|^{2}+\left|v^{\prime}(r)-i k v(r)\right|^{2}+(B(r) v(r), v(r))\right\}\right. \\
& \left.\quad+r^{2 \beta}|g(r)|^{2}\right]-\alpha r^{2 \eta}\left|v^{\prime}(r)-i k v(r)\right|^{2},
\end{aligned}
$$

since we have by (1.15) and (1.16)

$$
\begin{aligned}
& \left|v^{\prime \prime}(r)-B(r) v(r)+k^{2} v(r)\right|^{2} \leqq \delta_{1}\left[r ^ { - 2 - 2 \varepsilon } \left\{|v(r)|^{2}+\left|v^{\prime}(r)-i k v(r)\right|^{2}\right.\right. \\
& \left.\quad+(B(r) v(r), v(r))\}+|g(r)|^{2}\right]
\end{aligned}
$$

with a constant $\delta_{1}=\delta_{1}(k)>0$. We obtain from (1.14)

(1.26) $2 \operatorname{Re}\left(B(r) v(r), v^{\prime}(r)\right)=\frac{d}{d r}(B(r) v(r), v(r))-\left.\frac{d}{d r}(B(r) x, x)\right|_{x=v(r)}$

$$
\geqq \frac{d}{d r}(B(r) v(r), v(r))+\frac{e_{0}}{r}(B(r) v(r), v(r)) .
$$

(1.22), (1.23), (1.24) and (1.26) are combined to give

$$
\begin{aligned}
& \frac{d}{d r}\left(r^{\varepsilon} \psi(r)\left|v^{\prime}(r)-i k v(r)\right|^{2}\right) \\
& \quad \geqq \psi(r)\left\{\left(\varepsilon r^{-1+\varepsilon}-\frac{\delta_{1}}{\alpha} r^{-2-2 \varepsilon+2 \beta}-\alpha r^{2 \eta}\right)\left|v^{\prime}(r)-i k v(r)\right|^{2}\right.
\end{aligned}
$$




$$
\begin{aligned}
& +\left(e_{0} r^{-1+\varepsilon}-\frac{\delta_{1}}{\alpha} r^{-2-2 \varepsilon+2 \beta}\right)(B(r) v(r), v(r)) \\
& +r^{\varepsilon} \frac{d}{d r}(B(r) v(r), v(r))-\frac{\delta_{1}}{\alpha} r^{-2-2 \varepsilon+2 \beta}|v(r)|^{2} \\
& \left.-\frac{\delta_{1}}{\alpha} r^{2 \beta}|g(r)|^{2}\right\} .
\end{aligned}
$$

Putting $\eta=\frac{1}{2}(-1+\varepsilon), \quad \beta=\frac{1}{2}(1+\varepsilon)$ and $\alpha=\frac{1}{2} \varepsilon$, we integrate (1.27) from $r_{0}$ to $R\left(R \geqq r_{0}+2\right)$ to obtain

$$
\begin{aligned}
& R^{\varepsilon}\left|v^{\prime}(R)-i k v(R)\right|^{2} \\
& \geqq \int_{r_{0}}^{R} \psi(r)\left(\frac{\varepsilon}{2} r^{-1+\varepsilon}-\frac{2 \delta_{1}}{\varepsilon} r^{-1-\varepsilon}\right)\left|v^{\prime}(r)-i k v(r)\right|^{2} d r \\
& \quad+\int_{r_{0}}^{R} \psi(r)\left(\left(e_{0}-\varepsilon\right) r^{-1+\varepsilon}-\frac{2 \delta_{1}}{\varepsilon} r^{-1-\varepsilon}\right)(B(r) v(r), v(r)) d r \\
& \quad-\frac{2 \delta_{1}}{\varepsilon} \int_{r_{0}}^{R} \psi(r)\left(r^{-1-\varepsilon}|v(r)|^{2}+r^{1+\varepsilon}|g(r)|^{2}\right) d r \\
& \quad-\int_{r_{0}}^{r_{0}+1} r^{\varepsilon} \psi^{\prime}(r)(B(r) v(r), v(r)) d r
\end{aligned}
$$

where we have made use of the estimate

$$
\begin{aligned}
& \int_{r_{0}}^{R} r^{\varepsilon} \psi(r) \frac{d}{d r}(B(r) v(r), v(r)) d r \\
& =R^{\varepsilon}(B(R) v(R), v(R))-\int_{r_{0}}^{R}\left[\frac{d}{d r}\left(r^{\varepsilon} \psi(r)\right)\right](B(r) v(r), v(r)) d r \\
& \geqq-\int_{r_{0}}^{R} \varepsilon r^{-1+\varepsilon} \psi(r)(B(r) v(r), v(r)) d r \\
& \quad-\int_{r_{0}}^{r_{0}+1} r^{\varepsilon} \psi^{\prime}(r)(B(r) v(r), v(r)) d r .
\end{aligned}
$$

Now we take $r_{0}\left(\geqq b_{0}+1\right)$ so large that we have with a constant $\delta_{2}>0$

$$
\left\{\begin{array}{l}
\frac{\varepsilon}{2} r^{-1+\varepsilon}-\frac{2 \delta_{1}}{\varepsilon} r^{-1-\varepsilon} \geqq \delta_{2} r^{-1+\varepsilon} \\
\left(e_{0}-\varepsilon\right) r^{-1+\varepsilon}-\frac{2 \delta_{1}}{\varepsilon} r^{-1-\varepsilon} \geqq \delta_{2} r^{-1+\varepsilon}
\end{array}\right.
$$


for all $r \geqq r_{0}$. On the other hand, using Lemma 1.5 with $I_{1}=\left(r_{0}, r_{0}+1\right)$ and $I_{2}=\left(r_{0}-1, r_{0}+2\right)$, we obtain the following estimate with constants $K>0$ and $\delta_{3}>0$ :

$$
\begin{aligned}
& \int_{r_{0}}^{r_{0}+1} r^{\varepsilon} \psi^{\prime}(r)(B(r) v(r), v(r)) d r \\
& \quad \leqq\left(r_{0}+1\right)^{\varepsilon}\left(\max _{r_{0} \leqq r \leqq r_{0}+1} \psi^{\prime}(r)\right) \int_{r_{0}}^{r_{0}+1}(B(r) v(r), v(r)) d r \\
& \quad \leqq\left(r_{0}+1\right)^{\varepsilon}\left(_{r_{0} \leqq r \leqq r_{0}+1} \psi^{\prime}(\dot{r})\right) K \int_{r_{0}-1}^{r_{0}+2}\left(|v(r)|^{2}+|g(r)|^{2}\right) d r \\
& \quad<\delta_{3} \int_{r_{0}-1}^{R}\left(r^{-1-\varepsilon}|v(r)|^{2}+r^{1+\varepsilon}|g(r)|^{2}\right) d r,
\end{aligned}
$$

where we used (1.11). It follows from (1.28), (1.30) and (1.31) that

$$
\begin{aligned}
& \delta_{2} \int_{r_{0}+1}^{R} r^{-1+\varepsilon}\left\{\left|v^{\prime}(r)-i k v(r)\right|^{2}+(B(r) v(r), v(r))\right\} d r \\
& \leqq R^{\varepsilon}\left|v^{\prime}(R)-i k v(R)\right|^{2} \\
& \quad+\left(\frac{2 \delta_{1}}{\varepsilon}+\delta_{3}\right) \int_{r_{0}-1}^{R}\left(r^{-1-\varepsilon}|v(r)|^{2}+r^{1+\varepsilon}|g(r)|^{2}\right) d r
\end{aligned}
$$

Since $r^{-1+\varepsilon}\left|v^{\prime}(r)-i k v(r)\right|^{2}$ is integrable on $I_{0}$, we have

$$
R_{j}^{\varepsilon}\left|v^{\prime}\left(R_{j}\right)-i k v\left(R_{j}\right)\right|^{2} \rightarrow 0, \quad j \rightarrow \infty
$$

for some sequence $R_{j} \rightarrow \infty$. Thus we obtain (1.17) from (1.32). Q.E.D.

Lemma 1.6. Let us assume Assumption 1.1 and (a) and (c) of Assumption 1.2. Let $k \in \mathbf{C}^{+}$and let $v(r)$ be a radiative function for $\{L, k, l[g], 0\}$ with $v \in \operatorname{loc} H_{0}^{1, B}(\bar{I}, X) \cap H^{-1-\varepsilon}(I, X)$ and $g \in \operatorname{loc} H^{1, B}(\bar{I}, X)$ $\cap H^{1+\varepsilon}(I, X)$. Then there exists a constant $\delta>0$ such that

$$
\left\|v^{\prime}-i k v\right\|_{-1+\varepsilon}+\left\|B^{\frac{1}{2}} v\right\|_{-1+\varepsilon} \leqq \delta\left(\left\|v_{-1-\varepsilon}+\right\| g \|_{1+\varepsilon}\right),{ }^{12)}
$$

where $\delta$ depends only on $k$ and is bounded on any bounded set in $\mathbf{C}^{+}$.

12) Here and in the sequel we put \|\|$_{B, I}=\|\|_{\beta}$ and \|\|$_{B, I}=\|\|_{B}$ for the sake of simplicity. 
Proof. It follows from Assumption 1.1 that we can apply the regularity theorem of Jäger $[5]$ (Satz 3.1, p. 76) to see that $v \in C^{2}(I, D)$, $B v, C v \in C^{0}(I, X)$, and $v$ satisfies (1.15) for all $r \in I$. From Lemma 1.4 we obtain

$$
\begin{gathered}
\int_{r_{0}+1}^{\infty}(1+r)^{-1+\varepsilon}\left\{\left|v^{\prime}(r)-i k v(r)\right|^{2}+(B(r) v(r), v(r))\right\} d r \\
\leqq K_{1} \int_{r_{0}-1}^{\infty}\left\{(1+r)^{-1-\varepsilon}|v(r)|^{2}+r^{1+\varepsilon}|g(r)|^{2}\right\} d r
\end{gathered}
$$

with constants $r_{0} \geqq \rho_{1}+1$ and $K_{1}>0$. Since $v \in \operatorname{loc} H_{0}^{1, B}(\bar{I}, X)$, we can use the last statement of Lemma 1.5 with $I_{1}=\left(0, r_{0}+1\right)$ and $I_{2}$ $=\left(0, r_{0}+2\right)$ to obtain

$$
\begin{aligned}
& \int_{0}^{r_{0}+1}(1+r)^{-1+\varepsilon}\left\{\left|v^{\prime}(r)-i k v(r)\right|^{2}+(B(r) v(r), v(r))\right\} d r \\
& \quad \leqq K_{2} \int_{0}^{r_{0}+2}\left\{(1+r)^{-1-\varepsilon}|v(r)|^{2}+(1+r)^{1+\varepsilon}|g(r)|^{2}\right\} d r
\end{aligned}
$$

with a constant $K_{2}>0$. (1.34) follows from (1.35) and (1.36). Q.E.D.

\section{§2. The Uniqueness Theorem}

We shall show the uniqueness of the radiative function using arguments due to Jäger [5].

Lemma 2.1. Let $B(r)$ satisfy (a) and (b) of Assumption 1.1. Let $C(r)$ be a symmetric operator in $X$ for each $r \in I$ such that $C(r)$ satisfies (c) and (d) of Assumption 1.1. and

$$
|C(r) x| \leqq c\left(|x|+\left|B^{\frac{1}{2}}(r) x\right|\right), \quad(x \in D, r \in I)
$$

with a constant $c>0$. Let $v \in \operatorname{loc} H_{0}^{1, B}(\bar{I}, X)$ satisfy

$$
\left(\left(v,\left(L-\bar{k}^{2}\right) \varphi\right)\right)_{0}=((g, \varphi))_{0} \quad\left(\varphi \in C_{0}^{2, B}(I, X)\right)
$$

with $g \in \operatorname{loc} H^{1, B}(\bar{I}, X)$ and $k \in \mathbf{C}^{+}$, where

$$
L=-\frac{d^{2}}{d r^{2}}+B(r)+C(r)
$$


Then we have for all $r \in I$,

$$
\begin{aligned}
& \left|v^{\prime}(r)-i k v(r)\right|^{2} \\
& \quad=\left|v^{\prime}(r)+(\operatorname{Im} k) v(r)\right|^{2}+(\operatorname{Re} k)^{2}|v(r)|^{2} \\
& \quad+4(\operatorname{Re} k)^{2}(\operatorname{Im} k)\|v\|_{0,(0, r)}^{2}+2(\operatorname{Re} k) \operatorname{Im}((g, v))_{0,(0, r) .}
\end{aligned}
$$

Proof. As we have seen in the proof of Lemma 1.6, it follows from the regularity theorem of Jäger $[5]$ (p. 76) that

$$
\left\{\begin{array}{l}
v \in C^{2}(I, D) \quad \text { and } \quad B v, C v \in C^{0}(I, X) \\
\left(L-k^{2}\right) v(r)=g(r) \quad(r \in I) .
\end{array}\right.
$$

On the other hand we obtain from the fact that $v \in \operatorname{loc} H_{0}^{1, B}(I, X)^{13)}$

$$
\left\{\begin{array}{l}
v \in C^{0}(\bar{I}, X) \\
v(0)=0
\end{array}\right.
$$

From (2.5) and (2.6) we see that

$$
\begin{aligned}
& \int_{0}^{r}(g(t), \varphi(t)) d t \\
& =\int_{0}^{r}\left(\left(L-k^{2}\right) v(t), \varphi(t)\right) d t \\
& =\int_{0}^{r}\left\{\left(v^{\prime}(t), \varphi^{\prime}(t)\right)+\left(\left(B(t)+C(t)-k^{2}\right) v(t), \varphi(t)\right\} d t\right. \\
& \quad-\left(v^{\prime}(r), \varphi(r)\right)
\end{aligned}
$$

holds for any $\varphi \in C_{0}^{2, B}(I, X)$. Since $v \in \operatorname{loc} H_{0}^{1, B}(\bar{I}, X)$, for any $r>0$ there is a sequence $\left\{\varphi_{n}\right\}$ in $C_{0}^{2, B}(I, X)$ such that

$$
\left\{\begin{array}{l}
\left\|\varphi_{n}-v\right\|_{B,(0, r+1)} \rightarrow 0, \\
\varphi_{n}(t) \rightarrow v(t) \quad \text { in } X \quad(t \in[0, r+1])
\end{array}\right.
$$

as $n \rightarrow \infty$. Replacing $\varphi$ by $\varphi_{n}$ in (2.7) and letting $n \rightarrow \infty$, we have

13) Note that $H^{1, B}(I, X)$ is continuously imbedded in $C^{0}(\bar{I}, X)$. See Jäger [5], p. 69 . 


$$
\begin{aligned}
\int_{0}^{r}(g(t), v(t)) d t= & \int_{0}^{r}\left\{\left|v^{\prime}(t)\right|^{2}+\left(\left(B(t)+C(t)-k^{2}\right) v(t), v(t)\right)\right\} d t \\
& -\left(v^{\prime}(r), v(r)\right)
\end{aligned}
$$

Hence we obtain

$$
\begin{aligned}
& \operatorname{Im}\left(v^{\prime}(r), v(r)\right) \\
& \quad=-\operatorname{Im}((g, v))_{0,(0, r)}-2(\operatorname{Re} k)(\operatorname{Im} k)\|v\|_{0,(0, r)}^{2} .
\end{aligned}
$$

Using (2.10), we calculate $\left|v^{\prime}(r)-i k v(r)\right|^{2}$ as follows:

$$
\begin{aligned}
& \left|v^{\prime}(r)-i k v(r)\right|^{2} \\
& =\left|v^{\prime}(r)+(\operatorname{Im} k) v(r)\right|^{2}+(\operatorname{Re} k)^{2}|v(r)|^{2} \\
& \quad-2(\operatorname{Re} k) \operatorname{Im}\left(v^{\prime}(r), v(r)\right) \\
& =\left|v^{\prime}(r)+(\operatorname{Im} k) v(r)\right|^{2}+(\operatorname{Re} k)^{2}|v(r)|^{2} \\
& \quad+4(\operatorname{Re} k)^{2}(\operatorname{Im} k)|| v \|_{0,(0, r)}^{2}+2(\operatorname{Re} k) \operatorname{Im}((g, v))_{0,(0, r) .}
\end{aligned}
$$

Q.E.D.

Theorem 2.2. Let us assume Assumption 1.1 and (a) and (c) of Assumption 1.2. Let $l \in \mathscr{U}(I), k \in \mathbb{C}^{+}$and $u \in H^{1, B}(I, X)$ be given. Then the radiative function for $\{L, k, l, u\}$ is unique.

Proof. Let $v$ be a radiative function for $\{L, k, 0,0\}$, where $k \in \mathbf{C}^{+}$. What we want to show is that $v$ is identically zero.

We start with the relation

$$
\begin{aligned}
& \left|v^{\prime}(r)-i k v(r)\right|^{2}=\left|v^{\prime}(r)+(\operatorname{Im} k) v(r)\right|^{2} \\
& \quad+(\operatorname{Re} k)^{2}|v(r)|^{2}+4(\operatorname{Re} k)^{2}(\operatorname{Im} k)\|v\|_{0,(0, r)}^{2}
\end{aligned}
$$

which follows from Lemma 2.1.

If $\operatorname{Im} k>0$, then we obtain from (2.12) and the fact that $v^{\prime}-i k v$ $\in H^{-1+\varepsilon}(I, X)$

$$
0 \leqq\|v\|_{0,\left(0, r_{j}\right)}^{2} \leqq \frac{1}{4(\operatorname{Re} k)^{2}(\operatorname{Im} k)}\left|v^{\prime}\left(r_{j}\right)-i k v\left(r_{j}\right)\right|^{2} \rightarrow 0, j \rightarrow \infty
$$


for some sequence $r_{j} \rightarrow \infty$. Hence we have $\|v\|_{0}^{2}=0$, i.e., $v \equiv 0$.

Next let us assume that $\operatorname{Im} k=0$. Then we have from (2.12) and the radiation condition $v^{\prime}-i k v \in H^{-1+\varepsilon}(I, X)$

$$
\begin{aligned}
& \frac{\lim _{r \rightarrow \infty}}{}\left(\left|v^{\prime}(r)\right|^{2}+k^{2}|v(r)|^{2}\right) \\
& \quad=\frac{\lim _{r \rightarrow \infty}}{r \rightarrow v^{\prime}}(r)-\left.i k v(r)\right|^{2}=0 .
\end{aligned}
$$

By the regularity theorem of Jäger [5] (p. 76) and (1.8) we have

$$
\begin{aligned}
& \left\{\begin{array}{l}
v \in C^{2}(I, D) \\
\left|v^{\prime \prime}(r)-B(r) v(r)+k^{2} v(r)\right|^{2}=|C(r) v(r)|^{2}
\end{array}\right. \\
& \leqq 2 c_{2}^{2}(1+r)^{-2-2 \varepsilon}\left\{|v(r)|^{2}+(B(r) v(r), v(r))\right\} \quad(r \in I),
\end{aligned}
$$

where $c_{2}>0$ is given in (1.8). (2.14) and (2.15) enable us to apply Hilfssatz 1 of Jäger [3] (p. 66) on the growth property of solutions of the equation $\left(L-k^{2}\right) v=0$ to show that the carrier of $v$ is compact in $I$. Hence, using Satz 3 of Jäger [4] (p. 32), a unique continuation theorem for solutions of the equation $\left(L-k^{2}\right) v=0$, we see that $v \equiv 0$ on $I$.

Q.E.D.

\section{§3. The Existence Theorems}

This section is devoted to showing the existence of the radiative function $v$ for $\{L, k, l, u\}$, where $k \in \mathbb{C}, u \in H^{1, B}(I, X)$, and $l$ belongs to a subspace $\mathscr{U}_{1+\varepsilon}(I)$ of $\mathscr{U}(I)$. We shall first prove a priori estimates for radiative functions $v$ for $\{L, k, l, 0\}, k \in \mathbb{C}^{+}$and $l \in \mathscr{U}_{1+\varepsilon}(I)$ (Lemma 3.1 and Lemma 3.4). This corresponds to Satz 5.3 of Jäger [5]. But it seems that we have to modify its proof in order to obtain the a priori estimates needed in our case. Lemma 3.2 is necessary for this modification. Next we shall prove the existence theorems using our a priori estimates (Theorem 3.7 and Theorem 3.8). At the same time we shall see that the radiative function $v$ for $\{L, k, l, u\}$ depends continuously on $k, l$ and $u$. 
Lemma 3.1. Let us assume Assumptions 1.1 and 1.2. Let $K$ be $a$ compact set in $\mathbf{C}^{+}$. Let $k \in K$ and $g \in H^{1+\varepsilon}(I, X) \cap \operatorname{loc} H^{1, B}(\bar{I}, X)$. Let $v$ be a radiative function for $\{L, k, l[g], 0\}$ such that

$$
v \in \operatorname{loc} H_{0}^{1, B}(\bar{I}, X) \cap H^{-1-\varepsilon}(I, X) .
$$

Then we have

$$
\|v\|_{-1-\varepsilon}+\left\|v^{\prime}-i k v\right\|_{-1+\varepsilon}+\left\|B^{\frac{1}{2}} v\right\|_{-1+\varepsilon} \leqq \delta_{1}\|g\|_{1+\varepsilon} .
$$

with a constant $\delta_{1}>0$, where $\delta_{1}$ depends only on $K$ and $L$.

To prove this lemma we prepare

Lemma 3.2. Let $K, g, k$ and $v$ be as in Lemma 3.1. Then there exists a positive number $\alpha_{0}$ such that

$$
\int_{\rho}^{\infty}(1+r)^{-1-\varepsilon}|v(r)|^{2} d r \leqq \alpha_{0}\left(\|v\|_{-1-\varepsilon}^{2}+\|g\|_{1+\varepsilon}^{2}\right) \rho^{-\varepsilon}, \quad(\rho \geqq 1)
$$

where $\alpha_{0}$ depends only on $K$ and $L$.

Proof. From Lemma 2.1 we obtain

$$
(\operatorname{Re} k)^{2}|v(r)|^{2}+2(\operatorname{Re} k) \operatorname{Im}((g, v))_{0,(0, r)} \leqq\left|v^{\prime}(r)-i k v(r)\right|^{2},
$$

whence we have

$$
\begin{aligned}
|v(r)|^{2} \leqq & \frac{1}{(\operatorname{Re} k)^{2}}\left|v^{\prime}(r)-i k v(r)\right|^{2}+\frac{2}{|\operatorname{Re} k|} \int_{0}^{r}|g(t)||v(t)| d t \\
\leqq & \frac{1}{(\operatorname{Re} k)^{2}}\left|v^{\prime}(r)-i k v(r)\right|^{2} \\
& +\frac{2}{|\operatorname{Re} k|}\left\{\int_{0}^{r}(1+t)^{1+\varepsilon}|g(t)|^{2} d t\right\}^{\frac{1}{2}}\left\{\int_{0}^{r}(1+t)^{-1-\varepsilon}|v(t)|^{2} d t\right\}^{\frac{1}{2}} \\
\leqq & \frac{1}{(\operatorname{Re} k)^{2}}\left|v^{\prime}(r)-i k v(r)\right|^{2}+\frac{2}{|\operatorname{Re} k|}\|g\|_{1+\varepsilon}\|v\|_{-1-\varepsilon} .
\end{aligned}
$$

Multiplying both sides of (3.5) by $r^{-1-\varepsilon}$ and integrating from $\rho$ to $\infty$, we have 


$$
\begin{aligned}
\int_{\rho}^{\infty} r^{-1-\varepsilon}|v(r)|^{2} d r \leqq & \frac{1}{(\operatorname{Re} k)^{2}} \int_{\rho}^{\infty} r^{-1-\varepsilon}\left|v^{\prime}(r)-i k v(r)\right|^{2} d r \\
& +\frac{2}{\varepsilon|\operatorname{Re} k|}\|g\|_{1+\varepsilon}\|v\|_{-1-\varepsilon} \rho^{-\varepsilon} \\
\leqq & \frac{1}{(\operatorname{Re} k)^{2}} \rho^{-2 \varepsilon} \int_{\rho}^{\infty} r^{-1+\varepsilon}\left|v^{\prime}(r)-i k v(r)\right|^{2} d r \\
& +\frac{2}{\varepsilon|\operatorname{Re} k|}\|g\|_{1+\varepsilon}\|v\|_{-1-\varepsilon} \rho^{-\varepsilon} .
\end{aligned}
$$

(3.3) follows from (3.6) and Lemma 1.6.

Q.E.D.

Proof of Lemma 3.1. It follows from Lemma 1.6 that it is enough to show

$$
\|v\|_{-1-\varepsilon} \leqq \alpha\|g\|_{1+\varepsilon}
$$

with a constant $\alpha>0$ depending only on $K$ and $L$. Let us assume that (3.7) is false. Then for each positive integer $n$ we can find $k_{n} \in K$, $h_{n} \in \operatorname{loc} H^{1, B}(\bar{I}, X)$, and radiative functions $u_{n}$ for $\left\{L, k_{n}, l\left[h_{n}\right], 0\right\}$ such that

$$
\left\|u_{n}\right\|_{-1-\varepsilon}>n\left\|h_{n}\right\|_{1+\varepsilon}
$$

Since we see $\left\|u_{n}\right\|_{-1-\varepsilon}>0$ from (3.8), we obtain radiative functions $v_{n}$ $=\frac{u_{n}}{\left\|u_{n}\right\|_{-1-\varepsilon}}$ for $\left\{L, k_{n}, l\left[g_{n}\right], 0\right\}, g_{n}=\frac{h_{n}}{\left\|u_{n}\right\|_{-1-\varepsilon}}$, with

$$
\left\{\begin{array}{l}
\left\|v_{n}\right\|_{-1-\varepsilon}=1, \\
\left\|g_{n}\right\|_{1+\varepsilon}<\frac{1}{n} .
\end{array}\right.
$$

Let $\left\{k_{n_{m}}\right\}$ be a subsequence of $\left\{k_{n}\right\}$ satisfying

$$
k_{n_{m}} \longrightarrow k_{0}, \quad m \longrightarrow \infty
$$

with $k_{0} \in K$. Without loss of generality we can assume

$$
k_{n} \longrightarrow k_{0}, \quad n \longrightarrow \infty \text {. }
$$

In view of (3.9) we have for any $R \in I$ 


$$
\left\{\begin{array}{l}
\sup _{n}\left\|v_{n}\right\|_{0,(0, R+1)}<\infty, \\
\sup _{n}\left\|g_{n}\right\|_{0,(0, R+1)}<\infty .
\end{array}\right.
$$

Therefore it follows from Lemma 1.5 that

$$
\sup _{n}\left\|v_{n}\right\|_{B,(0, R)}<\infty
$$

for all $R>0$. Since for all $0<R<\infty$ the imbedding $H_{0}^{1, B}((0, R), X) \rightarrow$ $H^{0}((0, R), X)$ is compact by (b) of Assumption 1.2, we obtain a subsequence of $\left\{v_{n}\right\}$ which is a Cauchy sequence in $H^{0}((0, R), X)$ for all $R \in I$. Without loss of generality we can assume that $\left\{v_{n}\right\}$ itself is a Cauchy sequence in $H^{0}((0, R), X)$ for all $R \in I$. The sequence $\left\{v_{n}\right\}$ is a Cauchy sequence in $H_{0}^{1, B}((0, R), X)$ for all $R \in I$, too. In fact for each pair $(n, m) v_{n}-v_{m}$ is the radiative function for $\left\{L, k, l\left[g_{n m}\right], 0\right\}$, where

$$
g_{n m}=g_{n}-g_{m}-\left(k_{0}^{2}-k_{n}^{2}\right) v_{n}+\left(k_{0}^{2}-k_{m}^{2}\right) v_{m}
$$

and $k_{0}$ is given as in (3.11). From (3.9) and (3.11) we obtain $g_{n m} \rightarrow 0$, $n, m \rightarrow \infty$ in $H^{0}((0, R+1), X)$ for any $R>0$. Hence, noting that $\left\{v_{n}\right\}$ is a Cauchy sequence in $H^{0}((0, R+1), X)$, we can apply Lemma 1.5 to show

$$
\begin{aligned}
\left\|v_{n}-v_{m}\right\|_{B,(0, R)} \leqq & \beta\left(\left\|v_{n}-v_{m}\right\|_{0,(0, R+1)}+\left\|g_{n, m}\right\|_{0,(0, R+1)}\right) \\
& \longrightarrow 0, \quad n, m \longrightarrow \infty
\end{aligned}
$$

where $\beta>0$ depends only on $R, k$ and $L$. Therefore there exists $v \in \operatorname{loc} H_{0}^{1, B}(\bar{I}, X)$ satisfying

$$
v_{n} \longrightarrow v, \quad n \longrightarrow \infty
$$

both in $H_{0}^{1, B}((0, R), X)$ and in $H^{0}((0, R), X)$ for any $R \in I$.

Letting $n \rightarrow \infty$ in the relation

$$
\left(\left(v_{n},\left(L-\bar{k}_{n}^{2}\right) \varphi\right)\right)_{0}=\left(\left(g_{n}, \varphi\right)\right)_{0}, \quad\left(\varphi \in C_{0}^{2, B}(I, X)\right)
$$

we obtain from (3.16), (3.9) and (3.11) 


$$
\left(\left(v,\left(L-\bar{k}_{0}^{2}\right) \varphi\right)\right)_{0}=0 .
$$

Using (3.9), (3.16) and Lemma 1.6 we estimate $\left\|v^{\prime}-i k_{0} v\right\|_{-1+\varepsilon,(0, R)}$ as follows :

$$
\begin{aligned}
i \mid v^{\prime}-i k_{0} v \|_{-1+\varepsilon,(0, R)} & =\lim _{n \rightarrow \infty}\left\|v_{n}^{\prime}-i k_{0} v_{n}\right\|_{-1+\varepsilon,(0, R)} \\
& \leqq \sup _{n}\left\|v_{n}^{\prime}-i k_{0} v_{n}\right\|_{-1+\varepsilon} \\
& \leqq \delta \sup _{n}\left\{\left\|v_{n}\right\|_{-1-\varepsilon}+\left\|g_{n}\right\|_{1+\varepsilon}\right\} \\
& \leqq \delta \sup _{n}\left(1+\frac{1}{n}\right) \leqq 2 \delta,
\end{aligned}
$$

where $\delta>0$ is as in Lemma 1.6. Since the last member of (3.19) does not depend on $n$ and $R$, we have $v^{\prime}-i k_{0} v \in H^{-1+\varepsilon}(I, X)$, i.e., $v$ satisfies the radiation condition. Thus $v$ is a radiative function for $\left\{L, k_{0}, 0,0,\right\}$, and hence $v \equiv 0$ by Theorem 2.2 .

From Lemma 3.2 we obtain for $\rho \geqq 1$

$$
\begin{aligned}
\varlimsup_{n \rightarrow \infty}\left\|v_{n}\right\|_{-1-\varepsilon}^{2} & \leqq \lim _{n \rightarrow \infty}\left\|v_{n}\right\|_{-1-\varepsilon,(0, \rho)}^{2}+\sup _{n}\left\|v_{n}\right\|_{-1-\varepsilon,(\rho, \infty)}^{2} \\
& \leqq\|v\|_{-1-\varepsilon,(0, \rho)}^{2}+\alpha_{0} \rho^{-\varepsilon} \sup _{n}\left\{\left\|v_{n}\right\|_{-1-\varepsilon}^{2}+\left\|g_{n}\right\|_{1+\varepsilon}^{2}\right\} \\
& =0\left(\rho^{-\varepsilon}\right),
\end{aligned}
$$

where we have noted (3.9) and the fact $v \equiv 0$. Since $\rho \geqq 1$ is arbitrary, we obtain $\lim _{n \rightarrow \infty} \mid i v_{n} \|_{-1-\varepsilon}=0$, which contradicts the assumption that $\left\|v_{n}\right\|_{-1-\varepsilon}$ $=1$.

Q.E.D.

Now we introduce a subspace of $\mathscr{U}(I)$.

Definition 3.3. Let $\mathscr{U}_{1+\varepsilon}(I)$ be the set of all $l \in \mathscr{U}(I)$ such that

(3.21) $\|l\|_{1+\varepsilon}=\sup \left\{\left|<l,(1+r)^{\frac{1+\varepsilon}{2}} \varphi>\right| ; \varphi \in C_{0}^{2, B}(I, X),\|\varphi\|_{B}=1\right\}<\infty$. $\mathscr{U}_{1+\varepsilon}(I)$ is a Banach space with the norm \|\|$_{1+\varepsilon}$.

It is easy to see that we have

$$
\|l\| \leqq a_{0}\|l\|_{1+\varepsilon} \quad\left(l \in \mathscr{U}_{1+\varepsilon}(I)\right)
$$


with a constant $a_{0}>0$.

We shall show that the inequality (3.2) also holds for the radiative function for $\{L, k, l, 0\}$, where $l \in \mathscr{U}_{1+\varepsilon}(I)$.

Lemma 3.4. Let us assume Assumptions 1.1 and 1.2. Let $K$ be as in Lemma 3.1. Let $k \in K$ and $l \in \mathscr{U}_{1+\varepsilon}(I)$. Let $v$ be a radiative function for $\{L, k, l, 0\}$ such that $v \in H^{-1-\varepsilon}(I, X) \cap \operatorname{loc} H_{0}^{1, B}(\bar{I}, X)$.

Then there exists a constant $\delta_{2}>0$ such that

$$
\|v\|_{-1-\varepsilon}+\left\|v^{\prime}-i k v\right\|_{-1+\varepsilon}+\left\|B^{\frac{1}{2}} v\right\|_{-1+\varepsilon} \leqq \delta_{2}\|l\|_{1+\varepsilon},
$$

where $\delta_{2}$ depends only on $K$ and $L$.

To prove this lemma we need

Lemma 3.5. ${ }^{14)}$ Let $B(r)$ satisfy (a) of Assumption 1.1 and let $C(r)$ $(r \in I)$ be a symmetric operator in $X$ with the domain $\mathscr{D}(C(r))=D$ such that

$$
|C(r) x| \leqq c\left(|x|+\left|B^{\frac{1}{2}}(r) x\right|\right) \quad(r \in I, x \in D)
$$

with a constant $c>0$. Let $k_{0} \in \mathbf{C}^{+}$and $\operatorname{Im} k_{0}>0$. Let $l \in \mathscr{U}(I)$. Then the equation

$$
\left(\left(u,\left(L-\bar{k}_{0}^{2}\right) \varphi\right)\right)_{0}=\langle l, \varphi\rangle \quad\left(\varphi \in C_{0}^{2, B}(I, X)\right)
$$

has a unique solution $u$ in $H_{0}^{1, B}(I, X)$ with the estimate

$$
\|u\|_{B} \leqq \beta_{1}\|l\|
$$

where $\beta_{1}=\beta_{1}\left(k_{0}\right)>0$ is a constant. Further, if $l \in \mathscr{U}_{1+\varepsilon}(I)$, then we have $u \in H^{1+\varepsilon}(I, X)$ and

$$
\|u\|_{1+\varepsilon} \leqq \beta_{2}\|l\|_{1+\varepsilon}
$$

with a constant $\beta_{2}=\beta_{2}\left(k_{0}\right)>0$.

Proof. Let us define a bilinear form $\mathscr{B}_{L-k_{0}^{2}}[\cdot, \cdot]$ on $H_{0}^{1, B}(I, X)$

14) Cf. Jäger [5], Lemma 2.3 (p. 75) and the proof of Satz 5.3 (p. 86). 
$\therefore H_{0}^{1, B}(I, X)$ by

$$
\mathscr{B}_{L-k_{0}^{2}}\left[w_{1}, w_{2}\right]=\left(\left(w_{1}, w_{2}\right)\right)_{B}+\left(\left(\left(C(r)-k_{0}^{2}-1\right) w_{1}, w_{2}\right)\right)_{0} .
$$

Then we shall show

$$
d_{1}|| w\left\|_{B}^{2} \geqq\left|\mathscr{B}_{L-k_{0}^{2}}[w, w]\right| \geqq d_{2}\right\| w \|_{B}^{2} \quad\left(w \in H_{0}^{1, B}(I, X)\right)
$$

where $d_{j}=d_{j}\left(k_{0}\right)>0(j=1,2)$ are constants. Since $C_{0}^{2, B}(I, X)$ is dense in $H_{0}^{1, B}(I, X)$, and we have by integration by parts

$$
\mathscr{B}_{L-k_{0}^{2}}\left[\varphi_{1}, \varphi_{2}\right]=\left(\left(\varphi_{1},\left(L-\bar{k}_{0}^{2}\right) \varphi_{2}\right)\right)_{0} \quad\left(\varphi_{1}, \varphi_{2} \in C_{0}^{2}(I, X)\right),
$$

it is sufficient to show (3.29) that we show

$$
\left\{\begin{array}{l}
d_{1}\|\varphi\|_{B}^{2} \geqq\left|\left(\left(\varphi,\left(L-\bar{k}_{0}^{2}\right) \varphi\right)\right)_{0}\right|, \\
d_{2}\|\varphi\|_{B}^{2} \leqq\left|\left(\left(\varphi,\left(L-\bar{k}_{0}^{2}\right) \varphi\right)\right)_{0}\right|,
\end{array} \quad\left(\varphi \in C_{0}^{2, B}(I, X)\right)\right.
$$

Let us prove (3.31). From (3.24) we see that

$$
\|C \varphi\|_{0}^{2} \leqq \int_{I} c^{2}\left(|\varphi(r)|+\left|B^{\frac{1}{2}}(r) \varphi(r)\right|\right)^{2} d r \leqq 2 c\|\varphi\|_{B}^{2}
$$

whence follows for all $\varphi \in C_{0}^{2, B}(I, X)$

$$
\begin{aligned}
\left|\left(\left(\varphi,\left(L-\bar{k}_{0}^{2}\right) \varphi\right)\right)_{0}\right| & \leqq\|\varphi\|_{B}^{2}+\sqrt{2 c}\|\varphi\|_{B}\|\varphi\|_{0}+\left|k_{0}\right|^{2}\|\varphi\|_{0}^{2} \\
& \leqq\left(1+\sqrt{2 c}+\left|k_{0}\right|^{2}\right)\|\varphi\|_{B}^{2} .
\end{aligned}
$$

Thus we have shown the first inequality of (3.31) with $d_{1}=(1+\sqrt{2 c}$ $\left.+\left|k_{0}\right|^{2}\right)$. On the other hand we have

$$
\left|\left(\left(\varphi,\left(L-\bar{k}_{0}^{2}\right) \varphi\right)\right)_{0}\right|^{2}=\left\{\|\varphi\|_{B}^{2}+(((C-\lambda-1) \varphi, \varphi))_{0}\right\}^{2}+\mu^{2}\|\varphi\|_{0}^{4}
$$

where $\lambda=\operatorname{Re} k_{0}^{2}$ and $\mu=\operatorname{Im} k_{0}^{2} \neq 0$. Hence, using (3.32) again, we have

$$
\begin{aligned}
& \left|\left(\left(\varphi,\left(L-\bar{k}_{0}^{2}\right) \varphi\right)\right)_{0}\right|^{2} \geqq\|\varphi\|_{B}^{4}-2\|\varphi\|_{B}^{2}\left|(((C-\lambda-1) \varphi, \varphi))_{0}\right| \\
& \quad+(((C-\lambda-1) \varphi, \varphi))^{2}+\mu^{2}\|\varphi\|_{0}^{4} \\
& \quad \geqq(1-\alpha)\left\|_{\mid} \varphi\right\|_{B}^{4}-\left(\frac{1}{\alpha}-1\right)(((C-\lambda-1) \varphi, \varphi))_{0}^{2}+\mu^{2}\|\varphi\|_{0}^{4}
\end{aligned}
$$


with $\alpha>0$. Take $0<\alpha<1$ in (3.35). Then, noting that we obtain from (3.32)

$$
\begin{aligned}
& (((C-\lambda-1) \varphi, \varphi))_{0}^{2} \leqq\|(C-\lambda-1) \varphi\|_{0}^{2}\|\varphi\|_{0}^{2} \\
& \quad \leqq 2\left\{2 c^{2}+(|\lambda|+1)^{2}\right\}\|\varphi\|_{B}^{2}\|\varphi\|_{0}^{2} \\
& \quad \leqq\left\{2 c^{2}+(|\lambda|+1)^{2}\right\}\left(\beta\|\varphi\|_{B}^{4}+\frac{1}{\beta}\|\varphi\|_{0}^{4}\right) \quad(\beta>0)
\end{aligned}
$$

we arrive at

$$
\begin{aligned}
& \left|\left(\left(\varphi,\left(L-\bar{k}^{2}\right) \varphi\right)\right)_{0}\right|^{2} \geqq\left\{1-\alpha-\frac{1-\alpha}{\alpha} \beta c_{1}\right\}\|\varphi\|_{B}^{4} \\
& \quad+\left\{\mu^{2}-\frac{1-\alpha}{\alpha \beta} c_{1}\right\}\|\varphi\|_{0}^{4} \quad(0<\alpha<1, \beta>0),
\end{aligned}
$$

where we put $c_{1}=2 c^{2}+(|\lambda|+1)^{2}$. Putting $\beta=\frac{\alpha}{2 c_{1}}$ and taking $1-\alpha>0$ small enough, we obtain from (3.37)

$$
\left|\left(\left(\varphi,\left(L-\bar{k}^{2}\right) \varphi\right)\right)_{0}\right|^{2} \geqq \frac{1}{2}(1-\alpha)\|\varphi\|_{B}^{4}
$$

whence follows the second inequality of (3.31) with $d_{2}=\sqrt{\frac{(1-\alpha)}{2}}$.

Since (3.29) has been justified, we can make use of the Lax-Milgram theorem $^{15)}$ to show that there exists a unique solution of $u$ in $H_{0}^{1, B}(I, X)$ of the equation

$$
\mathscr{B}_{L-k_{0}^{2}}[u, w]=\langle l, w\rangle \quad\left(w \in H_{0}^{1, B}(I, X)\right)
$$

for $l \in \mathscr{U}(I)$. Since $\mathscr{B}_{L-k_{0}^{2}}[u, \varphi]=\left(\left(u,\left(L-\bar{k}_{0}^{2}\right) \varphi\right)\right)_{0}$ for $\varphi \in C_{0}^{2, B}(I, X)$, it follows from (3.38) that $u$ is a unique solution of (3.25). (3.29) and (3.39) are combined to give

$$
\|u\|_{B}^{2} \leqq \frac{1}{d_{2}}\left|\mathscr{B}_{L-k_{0}^{2}}[u, u]\right|=\frac{1}{d_{2}}|<l, u>| \leqq \frac{1}{d_{2}}\|l\|\|u\|_{B},
$$

which implies (3.26) with $\beta_{1}=\frac{1}{\sqrt{d_{2}}}$.

15) See, for example, Yosida [6], p. 92. 
Next let us show (3.27). Let $\phi \in C^{1}(\mathbf{R})$ such that $0 \leqq \psi(r) \leqq 1$, $0 \leqq\left|\psi^{\prime}(r)\right| \leqq 1$ and

$$
\phi(r)= \begin{cases}0 & \text { for } r \geqq 2, \\ 1 & \text { for } r \leqq \frac{1}{2} .\end{cases}
$$

For each $m=1,2, \ldots$ we define

$$
\left\{\begin{array}{l}
\psi_{m}(r)=(1+r)^{\frac{1+\varepsilon}{2}} \psi\left(\frac{r}{m}\right), \\
u_{m}=\psi_{m} u
\end{array}\right.
$$

where $u$ is the solution of the equation (3.38). Then we obtain from (3.39) and (3.28)

$$
\begin{aligned}
\mathscr{B}_{L-k_{0}}^{2} & {\left[u_{m}, u_{m}\right]=\left\|u_{m}\right\|_{B}^{2}+\left(\left(\left(C(r)-k_{0}^{2}-1\right) u_{m}, u_{m}\right)\right)_{0} } \\
= & \left(\left(\left(\psi_{m} u\right)^{\prime}, u_{m}^{\prime}\right)\right)_{0}+\left(\left(B^{2} u, B^{\frac{1}{2}} \psi_{m} u_{m}\right)\right)_{0} \\
& +\left(\left(\left(C(r)-k_{0}^{2}\right) u, \psi_{m} u_{m}\right)\right)_{0} \\
= & \mathscr{B}_{L-k_{0}^{2}}\left[u, \psi_{m} u_{m}\right]+\left(\left(\psi_{m}^{\prime} u, u_{m}^{\prime}\right)\right)_{0}-\left(\left(\psi_{m}^{\prime} u^{\prime}, u_{m}\right)\right)_{0} \\
= & <l, \psi_{m} u_{m}>+\left(\left(\psi_{m}^{\prime} u, u_{m}^{\prime}\right)\right)_{0}-\left(\left(\psi_{m}^{\prime} u^{\prime}, u_{m}\right)\right)_{0} .
\end{aligned}
$$

It follows from (3.43) and (3.29)

$$
\begin{aligned}
\left\|u_{m}\right\|_{B}^{2} \leqq & \frac{1}{d_{2}}\left|\mathscr{B}_{L-k_{0}}^{2}\left[u_{m}, u_{m}\right]\right| \\
\leqq & \frac{1}{d_{2}}\left\{\left|<l,(1+r)^{\frac{1+\varepsilon}{2}} \psi\left(\frac{r}{m}\right) u_{m}>\right|\right. \\
& \left.+\left\|\psi_{m}^{\prime} u\right\|_{0}\left\|u_{m}^{\prime}\right\|_{0}+l_{1}^{\prime} \psi_{m}^{\prime} u^{\prime}\left\|_{0}\right\| u_{m} \|_{0}\right\} \\
\leqq & \frac{1}{d_{2}}\left\{\|l\|_{1+\varepsilon}\left\|\phi\left(\frac{r}{m}\right) u_{m}\right\|_{B}+\left(\left\|\psi_{m}^{\prime} u\right\|_{0}+\left\|\psi_{m}^{\prime} u^{\prime}\right\|_{0}\right)\left\|u_{m}\right\|_{B}\right\} \\
\leqq & \frac{2}{d_{2}}\left\{\|l\|_{1+\varepsilon}+\left(\frac{1+\varepsilon}{2}+3\right)\|u\|_{B}\right\}\left\|u_{m}\right\|_{B},
\end{aligned}
$$

where we note $\left|\psi\left(\frac{r}{m}\right)\right| \leqq 1,\left|\frac{1}{m} \psi^{\prime}\left(\frac{r}{m}\right)\right| \leqq 1$ and 


$$
\left|\psi_{m}^{\prime}(r)\right| \leqq \frac{1+\varepsilon}{2}+3
$$

for any $r \in I$ and for any $m=1,2, \ldots$ Taking account of (3.26) and (3.22), we obtain from (3.44)

$$
\begin{aligned}
\left\|u_{m}\right\|_{B} & \leqq \frac{2}{d_{2}}\left\{\|l\|_{1+\varepsilon}+\left(\frac{1+\varepsilon}{2}+3\right) \beta_{1}\|l, l\|\right\} \\
& \leqq \frac{2}{d_{2}}\left\{1+\left(\frac{1+\varepsilon}{2}+3\right) \beta_{1} a_{0}\right\}\|l\|_{1+\varepsilon}
\end{aligned}
$$

which implies with $\frac{2}{d_{2}}\left(1+\left(\frac{1+\varepsilon}{2}+3\right) \beta_{1} a_{0}\right)=\beta_{2}$

$$
\left\|\psi\left(\frac{r}{m}\right) u\right\|_{1+\varepsilon}=\left\|\psi_{m} u\right\|_{0}=\left\|u_{m}\right\|_{0} \leqq\left\|u_{m}\right\|_{B} \leqq \beta_{2}\|l\|_{1+\varepsilon}
$$

Thus, letting $m \rightarrow \infty$ in (3.47), we obtain (3.27).

Q.E.D.

Proof of Lemma 3.4. Since $l \in \mathscr{U}_{1+\varepsilon}(I)$, it follows from Lemma 3.5 that the equation

$$
((g,(L+i) \varphi))_{0}=\langle l, \varphi\rangle \quad\left(\varphi \in C_{0}^{2, B}(I, X)\right)
$$

has a solution $g \in H_{0}^{1, B}(I, X) \cap H^{1+\varepsilon}(I, X)$. Put $w=g-v$, where $v$ is a radiative function for $\{L, k, l, 0\}$, i.e., $v$ satisfies

$$
\left(\left(v,\left(L-\bar{k}^{2}\right) \varphi\right)\right)_{0}=\langle l, \varphi\rangle \quad\left(\varphi \in C_{0}^{2, B}(I, X)\right)
$$

and

$$
\left\|v^{\prime}-i k v\right\|_{-1+\varepsilon}<\infty
$$

From (3.48) and (3.49) we see that

$$
\left(\left(w,\left(L-\bar{k}^{2}\right) \varphi\right)\right)_{0}=\left(k^{2}-i\right)((g, \varphi))_{0} .
$$

Noting $g \in H_{0}^{1, B}(I, X)$ and (3.50), we have

$$
\begin{aligned}
\left\|w^{\prime}-i k w\right\|_{-1+\varepsilon} & \leqq\left\|v^{\prime}-i k v\right\|_{-1+\varepsilon}+\left\|g^{\prime}-i k g\right\|_{-1+\varepsilon} \\
& \leqq\left\|v^{\prime}-i k v\right\|_{-1+\varepsilon}+\left\|g^{\prime}\right\|_{0}+|k|\|g\|_{0}<\infty
\end{aligned}
$$


Hence $w$ is a radiative function for $\left\{L, k,\left(k^{2}-i\right) l[g]\right\}$. We make use of Lemma 3.1 to obtain

$$
\|w\|_{-1-\varepsilon}+\left\|w^{\prime}-i k w\right\|_{-1+\varepsilon}+\left\|B^{\frac{1}{2}} w\right\|_{-1+\varepsilon} \leqq \delta_{1}\left(1+|k|^{2}\right)\|g\|_{1+\varepsilon}
$$

where $\delta_{1}=\delta_{1}(K)$ is given in (3.2). It is implied by (3.26) and (3.22) that

$$
\begin{aligned}
& \|g\|_{-1-\varepsilon}+\left\|g^{\prime}-i k g\right\|_{-1+\varepsilon}+\|\left. B^{\frac{1}{2}} g\right|_{-1+\varepsilon} ^{1} \\
& \leqq(1+|k|)\|g\|_{0}+\left\|g^{\prime}\right\|_{0}+\left\|B^{\frac{1}{2}} g\right\|_{0} \\
& \leqq(3+|k|)\|g\|_{B} \leqq(3+|k|)\|l\| \leqq(3+|k|) a_{0}\|l\|_{1+\varepsilon}
\end{aligned}
$$

Since $v=g+w,(3.23)$ follows from (3.53), (3.54) and (3.27). $\quad$ Q.E.D.

Lemma 3.6. Let us assume Assumptions 1.1 and 1.2. Let $k_{m} \in \mathbb{C}^{+}$, $l_{m} \in \mathscr{U}_{1+\varepsilon}(I)$ for each $m=1,2, \ldots$ Let $v_{m}, m=1,2, \ldots$ be radiative functions for $\left\{L, k_{m}, l_{m}, 0\right\}$ such that

$$
v_{m} \in H^{-1-\varepsilon}(I, X) \quad(m=1,2, \cdots) .
$$

Let us assume

$$
\left\{\begin{array}{l}
\lim _{m \rightarrow \infty} k_{m}=k, \\
\lim _{m \rightarrow \infty}\left\|l-l_{m}\right\|_{1+\varepsilon}=0
\end{array}\right.
$$

with $k \in \mathbb{C}^{+}$and $l \in \mathscr{U}_{1+\varepsilon}(I)$. Then there exists the radiative function $v$ for $\{L, k, l, 0\}$ satisfying

$$
v_{m} \rightarrow v \text { both in } H^{-1-\varepsilon}(I, X) \text { and in } \operatorname{loc} H_{0}^{1, B}(\bar{I}, X) \text { as } m \rightarrow \infty \text {. }
$$

Proof. As in the proof of Lemma 3.4 we put $v_{m}=g_{m}+w_{m}$, where $g_{m} \in H_{0}^{1, B}(I, X) \cap H^{1+\varepsilon}(I, X)$ is the solution of the equation

$$
\left(\left(g_{m},(L+i) \varphi\right)\right)_{0}=\left\langle l_{m}, \varphi>\quad\left(\varphi \in C_{0}^{2, B}(I, X)\right),\right.
$$

and $w_{m}$ is the radiative function for $\left\{L, k_{m},\left(k_{m}^{2}-i\right) l\left[g_{m}\right], 0\right\}$ for each $m=1,2, \ldots$. For each pair $(m, n)$ we have 


$$
\left(\left(g_{m}-g_{n},(L+i) \varphi\right)\right)_{0}=<l_{m}-l_{n}, \varphi>
$$

and hence we obtain, using Lemma 3.5,

$$
\left\{\begin{array}{l}
\left\|g_{m}-g_{n}\right\|_{B} \leqq \beta_{1}(\sqrt{i})\left\|l_{m}-l_{n}\right\| \rightarrow 0 \\
\left\|g_{m}-g_{n}\right\|_{1+\varepsilon} \leqq \beta_{2}(\sqrt{i})\left\|l_{m}-l_{n}\right\|_{1+\varepsilon} \rightarrow 0
\end{array}\right.
$$

as $m, n \rightarrow \infty$. We put $g=\lim _{m \rightarrow \infty} g_{m}$. Then $g \in H_{0}^{1, B}(I, X) \cap H^{1+\varepsilon}(I, X)$, and $g$ is the solution of equation (3.48).

Now we turn to the sequence $\left\{w_{m}\right\}$. Since the sequence $\left\{l_{m}\right\}$ is uniformly bounded in $\mathscr{U}_{1+\varepsilon}(I)$, it follows from Lemma 3.4 and Lemma 3.5 that the sequence $\left\{\left\|v_{m}\right\|_{-1-\varepsilon}+\left\|v_{m}^{\prime}-i k v_{m}\right\|_{-1+\varepsilon}+\left\|B^{2} v_{m}\right\|_{-1+\varepsilon}\right\},\left\{\left\|g_{m}\right\|_{B}\right\}$ and $\left\{\left\|g_{m}\right\|_{1+\varepsilon}\right\}$ are also uniformly bounded. Therefore, noting that $w_{m}=$ $v_{m}-g_{m}$, we obtain the uniform estimate

$$
\left\|w_{m}\right\|_{-1-\varepsilon}+\left\|w_{m}^{\prime}-i k_{m} w_{m}\right\|_{-1+\varepsilon}+\left\|B^{2} w_{m}\right\|_{-1+\varepsilon} \leqq \alpha \quad(m=1,2, \ldots)
$$

with a constant $\alpha>0$. From (3.61) we have

$$
\sup _{m=1,2, \ldots}\left\|w_{m}\right\|_{B,(0, R)}<\infty
$$

for any $R \in I$. Hence, proceeding as in the proof of Lemma 3.1, we obtain a subsequence $\left\{w_{m}\right\}$ of $\left\{w_{m}\right\}$ which converges to $w$ in $\operatorname{loc} H_{0}^{1, B}(\bar{I}, X)$. On the other hand, using Lemma 3.2 and the uniform boundedness of $\left\{\|g\|_{1+\varepsilon}\right\}$, we have uniformly with respect to $m$

$$
\begin{aligned}
\int_{\rho}^{\infty}(1+r)^{-1-\varepsilon}\left|w_{m}(r)\right|^{2} d r & \leqq \alpha_{0}\left(\left\|w_{m}\right\|_{-1-\varepsilon}^{2}+\left|k_{m}^{2}-i\right|^{2}\left\|g_{m}\right\|_{1+\varepsilon}\right) \rho^{-\varepsilon} \\
& =0\left(\rho^{-\varepsilon}\right) \quad(\rho \rightarrow \infty)
\end{aligned}
$$

where we have noted that $\left\{k_{m}\right\}$ is uniformly bounded and $w_{m}$ is a radiative function for $\left\{L, k,\left(k_{m}^{2}-i\right) l\left[g_{m}\right], 0\right\}$. It is implied by (3.63) and the convergence of $\left\{w_{m_{j}}\right\}$ in $\operatorname{loc} H_{0}^{1, B}(\bar{I}, X)$ that $w_{m}$, converges to $w$ in $H^{-1-\varepsilon}(I, X)$. Therefore, taking note of (3.61) and $k_{m} \rightarrow k, m \rightarrow \infty$, we see that $w$ is a radiative function for $\left\{L, k,\left(k^{2}-i\right) l[g], 0\right\}$ and we have

$$
w_{m,} \rightarrow w \quad(j \rightarrow \infty)
$$


both in $\operatorname{loc} H_{0}^{1, B}(\bar{I}, X)$ and $H^{-1-\varepsilon}(I, X)$.

Finally put $v_{m_{\jmath}}=g_{m_{\jmath}}+w_{m_{j}}$. Then we obtain from (3.60) and (3.64)

$$
v_{m} \rightarrow v \quad(j \rightarrow \infty)
$$

both in $\operatorname{loc} H_{0}^{1, B}(\bar{I}, X)$ and $H^{-1-\varepsilon}(I, X)$, where $v=g+w$ is a radiative function for $\{L, k, l, 0\}$. Since $v$ is unique by the uniqueness of the radiative function (Theorem 2.2), it follows from (3.65) that the original sequence $\left\{v_{m}\right\}$ itself converges to $v$ both in $H^{-1-\varepsilon}(I, X)$ and $\operatorname{loc} H_{0}^{1, B}(\bar{I}, X)$.

Q.E.D.

We can now prove the existence theorem of the radiative function for $\{L, k, l, 0\}$, where $k \in \mathbf{C}^{+}, l \in \mathscr{U}_{1+\varepsilon}(I)$.

Theorem 3.7. Let us assume Assumptions 1.1 and 1.2. Let $k \in \mathbf{C}^{+}$ and $l \in \mathscr{U}_{1+\varepsilon}(I)$. Then there exists a unique radiative function $v=v(\cdot, k, l)$ for $\{L, k, 0\}$ in $H^{-1-\varepsilon}(I, X)$. If $k$ belongs to a compact set $K$ in $\mathbf{C}^{+}$ then we have

$$
\|v\|_{-1-\varepsilon}+\left\|v^{\prime}-i k v\right\|_{-1+\varepsilon}+\left\|B^{\frac{1}{2}} v\right\|_{-1+\varepsilon} \leqq \delta_{2}\|l\|_{1+\varepsilon}
$$

with a constant $\delta_{2}>0$, depending only on $K$. Denote by $\Sigma_{0}$ the mapping

$$
\begin{aligned}
\Sigma_{0}: \mathbf{C}^{+} \times \mathscr{U}_{1+\varepsilon}(I) \ni(k, l) \\
\quad \rightarrow v(\cdot, k, l) \in H^{-1-\varepsilon}(I, X) \cap \operatorname{loc} H_{0}^{1, B}(\bar{I}, X) .
\end{aligned}
$$

Then $\Sigma_{0}$ is continuous as a mapping from $\mathbf{C}^{+} \times \mathscr{U}_{1+\varepsilon}(I)$ into $H^{-1-\varepsilon}(I, X)$ and is also continuous as a mapping from $\mathbf{C}^{+} \times \mathscr{U}_{1+\varepsilon}(I)$ into $\operatorname{loc} H_{0}^{1, B}(\bar{I}, X)$.

Proof. First assume that $\operatorname{Im} k>0$. Then from Lemma 3.5 we obtain a unique radiative function $v(\cdot, k, l)$ for $\{L, k, l, 0\}$ such that $v \in H^{1+\varepsilon}(I, X) \cap H_{0}^{1, B}(I, X)$. Next assume that $\operatorname{Im} k=0$. Then, putting for $m=1,2, \ldots$

$$
\left\{\begin{array}{l}
k_{m}=k+\frac{i}{m} \\
v_{m}=v_{m}\left(\cdot, k_{m}, l\right),
\end{array}\right.
$$


we see from Lemma 3.6 that the radiative function $v=v(\cdot, k, l)$ for $\{L, k, l, 0\}$ is obtained as $v=\lim _{m \rightarrow \infty} v_{m}$. The other statements follow from Lemma 3.4 and Lemma 3.6.

Q.E.D.

Finally we prove the existence of the radiative function for $\{L, k, l$, $u\}$.

Let $v=v(\cdot, k, l, u)$ be a radiative function for $\{L, k, l, u\}$, where $k \in \mathbb{C}^{+}, l \in \mathscr{U}_{1+\varepsilon}(I)$ and $u \in H^{1, B}(I, X)$. We define $l_{1} \in \mathscr{U}_{1+\varepsilon}(I)$ by

$$
\left\langle l_{1}, \varphi>=<l, \varphi>-\left(\left(\psi u,\left(L-\bar{k}^{2}\right) \varphi\right)\right)_{0} \quad\left(\varphi \in C_{0}^{2, B}(I, X)\right),\right.
$$

where $\psi \in C^{1}(I), 0 \leqq \psi \leqq 1$ and

$$
\psi(r)= \begin{cases}1 & (0<r \leqq 1), \\ 0 & (r \geqq 2) .\end{cases}
$$

Then it is easy to see that $v_{0}=v-\psi u$ is a radiative function for $\left\{L, k, l_{1}, 0\right\}$. Thus we can reduce the equation with the boundary value $v(0)=u(0)$ to the equation with the boundary value $v_{0}(0)=0$. Therefore, noting that $l_{1}=l_{1}(u)$ is a $\mathscr{U}_{1+\varepsilon}(I)$-valued continuous function on $H^{1, B}(I, X)$, we obtain from Theorem 3.7 the following

Theorem 3.8. Let us assume Assumptions 1.1 and 1.2. Let $k \in \mathbf{C}^{+}$, $l \in \mathscr{U}_{1+\varepsilon}(I)$ and $u \in H^{1, B}(I, X)$. Then there exists a unique radiative function $v=v(\cdot, k, l, u)$ for $\{L, k, l, u\}$ in $H^{-1-\varepsilon}(I, X)$. Denote by $\Sigma$ the mapping

$$
\begin{aligned}
\Sigma & : \mathbb{C}^{+} \times \mathscr{U}_{1+\varepsilon}(I) \times H^{1, B}(I, X) \ni(k, l, u) \\
& \rightarrow v(\cdot, k, l, u) \in H^{-1-\varepsilon}(I, X) \cap \operatorname{loc} H^{1, B}(\bar{I}, X) .
\end{aligned}
$$

Then $\Sigma$ is continuous as a mapping from $\mathbb{C}^{+} \times \mathscr{U}_{1+\varepsilon}(I) \times H^{1, B}(I, X)$ into $H^{-1-\varepsilon}(I, X)$ and is also continuous as a mapping from $\mathbb{C}^{+} \times \mathscr{U}_{1+\varepsilon}(I)$ $\times H^{1, B}(I, X)$ into $\operatorname{loc} H^{1, B}(\bar{I}, X)$.

\section{$\S 4$. The Dependency of Radiative Functions on $C(r)$}

Let $C_{m}(r), m=1,2, \ldots$, be a sequence of operator-valued functions on 
I. Let $C(r)$ be as above. In this section we study the relations between radiative functions for $L$ and radiative functions for $L_{m}=-\frac{d^{2}}{d r^{2}}+B(r)$ $+C_{m}(r)$ when $C_{m}(r) \rightarrow C(r)$ as $m \rightarrow \infty$.

Assumption 4.1. (a) For each $r \in I \quad C_{m}(r)$ is a symmetric operator in $X$ with $\mathscr{D}\left(C_{m}(r)\right)=D$ such that $C_{m}(r) x \in C^{1}(I, X)$ for any $x \in D$. Moreover for any compact inlerval $M$ in $I$ there exists a constant $c^{(m)}(M)$ $>0$ such that

$$
\left|\frac{d}{d r} C_{m}(r) x\right| \leqq c^{(m)}(M)\left(|x|+\left|B^{\frac{1}{2}}(r) x\right|\right)
$$

holds for any $x \in D$ and any $r \in M$.

(b) There exists a constant $c_{0}>0$ such that

$$
\left|C_{m}(r) x\right| \leqq c_{0}(1+r)^{-1-\varepsilon}\left(|x|+\left|B^{\frac{1}{2}}(r) x\right|\right) \quad(x \in D, r \in I)
$$

for any $m=1,2, \ldots$, where $c_{0}$ does not depend on $m$, and $0<\varepsilon<1$ is as given in (1.8).

(c) We have

$$
\lim _{m \rightarrow \infty}\left|C(r) x-C_{m}(r) x\right|=0
$$

for any $x \in D$ and any $r \in I$.

Since $C_{m}$ is assumed to satisfy (a) and (b) of Assumption 4.1 for each $m=1,2, \ldots, C_{m}(r)$ is so smooth and tends to zero at $r=\infty$ so rapidly that the results of $\S 2$ and $\S 3$ can be applied to the operator

$$
L_{m}=-\frac{d^{2}}{d r^{2}}+B(r)+C_{m}(r)
$$

i.e., there exists a unique radiative function $v_{m}(r, k, l, u)$ for $\left\{L_{m}, k, l, u\right\}$, where $(k, l, u) \in \mathbb{C}^{+} \times \mathscr{U}_{1+\varepsilon}(I) \times H^{1, B}(I, X)$.

Theorem 4.2. Let $B(r)$ and $C(r)$ satisfy Assumptions 1.1 and 1.2 . Let $C_{m}(r), m=1,2, \ldots$, satisfy Assumption 4.1. Let $K$ be a compact set such that $K \subset \mathrm{C}^{+}$and let $v_{m}=v_{m}\left(r, k_{m}, l_{m}\right), m=1,2, \ldots$, be the radiative 
function for $\left\{L_{m}, k_{m}, l_{m}, 0\right\}$, where $k_{m} \in K$ and $l_{m} \in \mathscr{U}_{1+\varepsilon}(I)$. Then there exists a constant $\delta_{0}>0$ such that

$$
\left\|v_{m}\right\|_{-1-\varepsilon}+\left\|v_{m}^{\prime}-i k_{m} v_{m}\right\|_{-1+\varepsilon}+\left\|B^{\frac{1}{2}} v_{m}\right\|_{-1+\varepsilon} \leqq \delta_{0}\left\|l_{m}\right\|_{1+\varepsilon}
$$

$\delta_{0}$ depends only on $K$.

Proof. Denote by $g_{m}$ the radiative function for $\left\{L_{m}, \sqrt{i}, l_{m}, 0\right\}$. We see from Lemma 3.5 that $g_{m} \in H^{1+\varepsilon}(I, X)$ for each $m=1,2, \ldots$. We denote by $w_{m}$ the radiative function for $\left\{L_{m}, k_{m},\left(k_{m}^{2}-i\right) l\left[g_{m}\right], 0\right\}$. Obviously we have $v_{m}=g_{m}+w_{m}$. Proceeding as in the proof of Lemma 3.5, from (4.2) we obtain uniformly for $m=1,2, \ldots$,

$$
\alpha\|\varphi\|_{B}^{2} \geqq\left|\left(\left(\varphi,\left(L_{m}+i\right) \varphi\right)\right)_{0}\right| \geqq \beta\|\varphi\|_{B}^{2} \quad\left(\varphi \in C_{0}^{2, B}(I, X)\right),
$$

with constants $\alpha, \beta>0$, whence follows that we obtain uniformly for $m=1,2, \ldots$

$$
\left\{\begin{array}{l}
\left\|g_{m}\right\|_{B} \leqq \eta_{0}\left\|l_{m}\right\|, \\
\left\|g_{m}\right\|_{1+\varepsilon} \leqq \eta_{0}\left\|l_{m}\right\|_{1+\varepsilon}
\end{array}\right.
$$

with a constant $\eta_{0}>0$. Re-examining the proof of Lemma 1.6, we can see from (4.2) that we obtain uniformly for $m=1,2, \ldots$

$$
\left\|w_{m}^{\prime}-i k w_{m}\right\|_{-1+\varepsilon}+\left\|B^{\frac{1}{2}} w_{m}\right\|_{-1+\varepsilon} \leqq \eta_{1}\left(\left\|w_{m}\right\|_{-1-\varepsilon}+\left\|g_{m}\right\|_{1+\varepsilon}\right),
$$

with a constant $\eta_{1}=\eta_{1}(K)>0$. Finally, proceeding as in the proof of Lemma 3.1 , we can show by reduction to absurdity that we have uniformly for $m=1,2, \ldots$

$$
\left\|w_{m}\right\|_{-1-\varepsilon} \leqq \eta_{2}\left\|g_{m}\right\|_{1+\varepsilon}
$$

with a positive constant $\eta_{2}=\eta_{2}(K)$. Thus we have (4.5) from (4.7), (4.8), (4.9) and (3.22) as follows:

$$
\begin{aligned}
& \left\|v_{m}\right\|_{-1-\varepsilon}+\left\|v_{m}^{\prime}-i k_{m} v_{m}\right\|_{-1+\varepsilon}+\left\|B^{\frac{1}{2}} v_{m}\right\|_{-1+\varepsilon} \\
& \leqq\left\|w_{m}\right\|_{-1-\varepsilon}+\left\|w_{m}^{\prime}-i k_{m} w_{m}\right\|_{-1+\varepsilon}+\left\|B^{\frac{1}{2}} w_{m}\right\|_{-1+\varepsilon} \\
& \quad+\left\|g_{m}\right\|_{0}+\left\|g_{m}^{\prime}\right\|_{0}+\left|k_{m}\right|\left\|g_{m}\right\|_{0}+\left\|B^{\frac{1}{2}} g_{m}\right\|_{0}
\end{aligned}
$$




$$
\begin{aligned}
& \leqq\left\|w_{m}\right\|_{-1-\varepsilon}+\eta_{1}\left(\left\|w_{m}\right\|_{-1-\varepsilon}+\left\|g_{m}\right\|_{1+\varepsilon}\right)+\left(3+\left|k_{m}\right|\right)\left\|g_{m}\right\|_{B} \\
& \leqq\left\{\eta_{2}+\eta_{1}\left(1+\eta_{2}\right)\right\}\left\|g_{m}\right\|_{1+\varepsilon}+\left(3+\left|k_{m}\right|\right)\left\|g_{m}\right\|_{B} \\
& \leqq\left[\left\{\eta_{2}+\eta_{1}\left(1+\eta_{2}\right)\right\}+(3+T) a_{0}\right]\left\|l_{m}\right\|_{1+\varepsilon} \quad(m=1,2, \ldots),
\end{aligned}
$$

where we put $T=\sup _{m=1,2}\left|k_{m}\right|$, and $a_{0}$ is given as in (3.22). Q.E.D.

Theorem 4.3. Let $B(r)$ and $C(r)$ satisfy Assumptions 1.1 and 1.2 . Let $C_{m}(r), m=1,2, \ldots$, satisfy Assumption 4.1.

(i) Let $k_{m} \in \mathbf{C}^{+}$and $l_{m} \in \mathscr{U}_{1+\varepsilon}(I)$ such that

$$
\begin{aligned}
& \mid \lim _{m \rightarrow \infty} k_{m}=k \\
& \mid \lim _{m \rightarrow \infty}\left\|l-l_{m}\right\|_{1+\varepsilon}=0
\end{aligned}
$$

with $k \in \mathbf{C}^{+}$and $l \in \mathscr{U}_{1+\varepsilon}(I)$. Denote by $v_{m}\left(\cdot, k_{m}, l_{m}\right)$ the radiative function for $\left\{L_{m}, k_{m}, l_{m}, 0\right\}$ for each $m=1,2, \ldots$. Then we have

$$
v_{m}\left(\cdot, k_{m}, l_{m}\right) \rightarrow v(\cdot, k, l)
$$

both in $H^{-1-\varepsilon}(I, X)$ and in $\operatorname{loc} H_{0}^{1, B}(\bar{I}, X)$, where $v(\cdot, k, l)$ is the radiative function for $\{L, k, l, 0\}$.

(ii) Let $K$ be a compact set in $\mathbf{C}^{+}$and let $M$ be a compact metric space. For each $m=1,2, \ldots, l_{m}(k, s)$ is assumed to be a $\mathscr{U}_{1+\varepsilon}(I)$-valued, continuous function on $K \times M$ such that

$$
\lim _{m \rightarrow \infty}\left\|l(k, s)-l_{m}(k, s)\right\|_{1+\varepsilon}=0
$$

uniformly on $K \times M$ with a $\mathscr{U}_{1+\varepsilon}(I)$-valued, continuous function $l(k, s)$ on $K \times M$. Denote by $v_{m}(\cdot, k, s)$ the radiative function for $\left\{L_{m}, k, l_{m}(k, s), 0\right\}$. Then we have

$$
\lim _{m \rightarrow \infty} v_{m}(\cdot, k, s)=v(\cdot, k, s)
$$

both in $H^{-1-\varepsilon}(I, X)$ and in $\operatorname{loc} H_{0}^{1, B}(\bar{I}, X)$ uniformly on $K \times M$, where $v(\cdot, k, s)$ is the radiative function for $\{L, k, l(k, s), 0\}$.

Proof. First let us prove (i). Let $g_{m}$ be the radiative function for 
$\left\{L, \sqrt{i}, l_{m}, 0\right\}$ and let $w_{m}$ be the radiative function for $\left\{L, k,\left(k_{m}^{2}-i\right) l\left[g_{m}\right]\right.$, $0\}$. Then we have $v_{m}=g_{m}+w_{m}$. Similarly we have $v=g+w$, where $g$ is the radiative function for $\{L, \sqrt{i}, l, 0\}$ and $w$ is the radiative function for $\left\{L, k,\left(k^{2}-i\right) l[g], 0\right\}$. It follows from Lemma 3.5 and the regularity theorem of Jäger [5] that $g, g_{m} \in H^{1+\varepsilon}(I, X) \cap H_{0}^{1, B}(I, X) \cap C^{2}(I, D)$. We can show that

$$
\lim _{m \rightarrow \infty}\left\|\left(C-C_{m}\right) g\right\|_{1+\varepsilon}=0 .
$$

In fact we obtain from (4.3) and the fact that $g(r) \in D$

$$
\lim _{m \rightarrow \infty}\left|\left(C(r)-C_{m}(r)\right) g(r)\right|=0 \quad(r \in I),
$$

and also obtain from (1.8) and (4.2)

$$
\begin{aligned}
& \left|\left(C(r)-C_{m}(r)\right) g(r)\right|^{2} \\
& \quad \leqq\left[\left(c_{2}+c_{0}\right)(1+r)^{-1-\varepsilon}\left(|g(r)|+\left|B^{\frac{1}{2}}(r) g(r)\right|\right)\right]^{2} \\
& \quad \leqq 2\left(c_{2}+c_{0}\right)^{2}(1+r)^{-2-2 \varepsilon}\left(|g(r)|^{2}+\left|B_{2}^{1}(r) g(r)\right|^{2}\right) \\
& \quad \in L^{1}\left(I,(1+r)^{1+\varepsilon} d r\right) .
\end{aligned}
$$

(4.15) directly follows from (4.16) and (4.17). Noting that $g-g_{m}$ satisfies the equation

$$
\begin{aligned}
& \left(\left(g-g_{m},(L+i) \varphi\right)\right)_{0}=\left\langle l-l_{m}, \varphi>+\left(\left(\left(C-C_{m}\right) g, \varphi\right)\right)_{0}\right. \\
& \left(\varphi \in C_{0}^{2, B}(I, X)\right) \text {, }
\end{aligned}
$$

We see from (4.7) and (4.15) that

$$
\left\{\begin{array}{l}
\left\|g-g_{m}\right\|_{B} \leqq \eta_{0}\left\{\left\|l-l_{m}\right\|+\left\|\left(C-C_{m}\right) g\right\|_{0}\right\} \rightarrow 0, \\
\left\|g-g_{m}\right\|_{1+\varepsilon} \leqq \eta_{0}\left\{\left\|l-l_{m}\right\|_{1+\varepsilon}+\left\|\left(C-C_{m}\right) g\right\|_{1+\varepsilon}\right\} \rightarrow 0
\end{array}\right.
$$

as $m \rightarrow \infty$. Using (4.19) and Theorem 4.2, we can proceed as in the proof of Lemma 3.6 to show that the sequence $w_{m}$ converges to $w$ both in $H^{-1-\varepsilon}(I, X)$ and in $\operatorname{loc} H_{0}^{1, B}(\bar{I}, X)$. Thus we have shown that $v_{m}=g_{m}$ $+w_{m}$ converges to $v=g+w$ both in $H^{-1-\varepsilon}(I, X)$ and in $\operatorname{loc}_{0}^{1, B}(\bar{I}, X)$ which completes the proof of (i). 
Next let us prove (ii). It follows from (i) that for each pair $(k, s)$ $\in K \times M \quad v_{m}(\cdot, k, s)$ converges to $v(\cdot, k, s)$ both in $H^{-1-\varepsilon}(I, X)$ and in loc $H_{0}^{1, B}(\bar{I}, X)$. Assume that the convergence of $v_{m}$ in $H^{-1-\varepsilon}(I, X)$ is not uniform on $K \times M$. Then there exists $\varepsilon_{0}>0$ and the set of positive integers $\left\{m_{j}\right\}_{j=1}^{\infty}$ and $\left(k_{j}, s_{j}\right) \in K \times M$ such that $m_{j} \rightarrow \infty$ as $j \rightarrow \infty$ and

$$
\left\|v\left(\cdot, k_{j}, s_{j}\right)-v_{m_{j}}\left(\cdot, k_{j}, s_{j}\right)\right\|_{-1-\varepsilon} \geqq \varepsilon_{0} .
$$

Since the set $\left\{\left(k_{j}, s_{j}\right) \mid j=1,2, \ldots\right\}$ has at least an accumulating point $\left(k_{0}, s_{0}\right) \in K \times M$, we can assume $k_{j} \rightarrow k_{0}$ and $s_{j} \rightarrow s_{0}$ without loss of generality. Then, using the continuity of $l(k, s)$ and the uniform convergence of $l_{m}(k, s)$, we obtain

$$
\begin{aligned}
& \left\|l\left(k_{0}, s_{0}\right)-l_{m_{j}}\left(k_{j}, s_{j}\right)\right\|_{1+\varepsilon} \\
& \quad \leqq\left\|l\left(k_{0}, s_{0}\right)-l\left(k_{j}, s_{j}\right)\right\|_{1+\varepsilon}+\left\|l\left(k_{j}, s_{j}\right)-l_{m_{j}}\left(k_{j}, s_{j}\right)\right\|_{1+\varepsilon} \rightarrow 0, \\
& j \rightarrow \infty .
\end{aligned}
$$

Therefore it follows from (i) that

$$
\left\|v\left(\cdot, k_{0}, s_{0}\right)-v_{m_{j}}\left(\cdot, k_{j}, s_{j}\right)\right\|_{-1-\varepsilon} \rightarrow 0, \quad j \rightarrow \infty .
$$

On the other hand we obtain from Lemma 3.6

$$
\left\|v\left(\cdot, k_{0}, s_{0}\right)-v\left(\cdot, k_{j}, s_{j}\right)\right\|_{-1-\varepsilon} \rightarrow 0, \quad j \rightarrow \infty .
$$

(4.22) and (4.23) are combined to give $\left\|v\left(\cdot, k_{j}, s_{j}\right)-v_{m_{j}}\left(\cdot, k_{j}, s_{j}\right)\right\|_{-1-\varepsilon} \rightarrow 0$, $j \rightarrow \infty$, which contradicts (4.20). Hence $v_{m}(\cdot, k, s)$ converges to $v(\cdot, k, s)$ in $H^{-1-\varepsilon}(I, X)$ uniformly for $(k, s) \in K \times M$. Similarly we can show that $v_{m}(\cdot, k, s)$ converges to $v(\cdot, k, s)$ in $\operatorname{loc}_{0}^{1, B}(\bar{I}, X)$ uniformly for $(k, s)$ $\in K \times M$.

Q.E.D.

By an argument similar to the one used in obtaining Theorem 3.8 from Theorem 3.7, we can show the following

Theorem 4.4. Let $B(r), C(r)$ and $C_{m}(r), m=1,2, \ldots$, be as in Theorem 4.3. Let $u \in H^{1, B}(I, X)$.

(i) Let $k_{m} \in \mathbf{C}^{+}$and $l_{m} \in \mathscr{U}_{1+\varepsilon}(I)$ satisfy (4.11). Denote by $v_{m}\left(\cdot, k_{m}\right.$, $\left.l_{m}, u\right)$ the radiative function for $\left\{L_{m}, k_{m}, l_{m}, u\right\}$ for each $m=1,2, \ldots$ 
Then we have $v_{m}\left(\cdot, k_{m}, l_{m}, u\right) \rightarrow v(\cdot, k, l, u), m \rightarrow \infty$, both in $H^{-1-\varepsilon}(I, X)$ and in $\operatorname{loc} H^{1, B}(\bar{I}, X)$, where $v(\cdot, k, l, u)$ is the radiative function for $\{L, k, l, u\}$.

(ii) Let $k, M, l_{m}(k, s)$ and $l(k, s)$ be as in (ii) of Theorem 4.3. Let (4.13) be satisfied. Then we have

$$
\lim _{m \rightarrow \infty} v_{m}\left(\cdot, k_{m}, s_{m}, u\right)=v(\cdot, k, s, u)
$$

both in $H^{-1-\varepsilon}(I, X)$ and in loc $H^{1, B}(\bar{I}, X)$ uniformly on $K \times M$, where $v_{m}\left(\cdot, k_{m}, s_{m}, u\right)$ and $v(\cdot, k, s, u)$ are the radiative functions for $\left\{L_{m}, k_{m}\right.$, $\left.l_{m}(k, s), u\right\}$ and $\{L, k, l(k, s), u\}$, respectively.

\section{§. The Schrödinger Operator in $\mathbf{R}^{n}(n \geqq 3)$}

In this section we apply the results obtained in the preceding sections to the Schrödinger operator in $\mathbf{R}^{n}(n \geqq 3)$.

Let $X=L^{2}\left(S^{n-1}\right), S^{n-1}$ being $(n-1)$-sphere. We define a unitary operator $U$ from $L^{2}\left(\mathbf{R}^{n}\right)$ onto $H^{0}(I, X)$ by

$$
(U F)(r)=r^{\frac{n-1}{2}} F(r \omega) \quad\left(F(y) \in L^{2}\left(\mathbf{R}^{n}\right)\right),
$$

where $r=|y|$ and $\omega=\frac{y}{r} \in S^{n-1}$.

Let us consider the Laplacian on $\mathbf{R}^{n}$

$$
-\Delta F(y)=-\sum_{j=1}^{n} \frac{\partial^{2} F}{\partial y_{j}^{2}} .
$$

We denote by $H_{0}$ the restriction of $-\Delta$ to $C_{0}^{\infty}\left(\mathbf{R}^{n}\right)$, i.e.,

$$
\left\{\begin{array}{l}
\left.\mathscr{D}\left(H_{0}\right)=C_{0}^{\infty}\left(\mathbf{R}^{n}\right),{ }^{16}\right) \\
H_{0} \Phi=-\Delta \Phi
\end{array}\right.
$$

As is well known, we have for $\Phi \in C_{0}^{\infty}\left(\mathbf{R}^{n}\right)$

$$
U H_{0} \Phi=L_{0} U \Phi
$$

16) $C_{0}^{\infty}\left(\mathbf{R}^{n}\right)$ is the set of all infinitely continuously differentiable functions on $\mathbf{R}^{n}$ with compact carrier. 
where

$$
\left\{\begin{array}{l}
L_{0}=-\frac{d^{2}}{d r^{2}}+B(r) \\
\mathscr{D}(B(r))=D=\mathscr{D}\left(\Lambda_{n}\right), \\
B(r)=\frac{1}{r^{2}}\left(-\Lambda_{n}+\frac{(n-1)(n-3)}{4}\right),
\end{array}\right.
$$

and $\Lambda_{n}$ is the Laplace-Beltrami operator on $S^{n-1}$. As is well-known $-\Lambda_{n}$ is a non-negative, self-adjoint operator in $L^{2}\left(S^{n-1}\right)$, and hence we can easily see that $B(r)$ satisfies (a) and (b) of Assumptions 1.1 and 1.2. We obtain from (5.4)

$$
\left\{\begin{array}{l}
U \mathscr{D}_{L^{2}}^{1}\left(\mathbf{R}^{n}\right)=H_{0}^{1, B}(I, X)^{17)} \\
\|F\|_{(1)}=\|U F\|_{B} \quad\left(F \in \mathscr{D}_{L^{2}}^{1}\left(\mathbf{R}^{n}\right)\right) .
\end{array}\right.
$$

Let $\mathscr{V}\left(R^{n}\right)$ be the set of all linear continuous functionals $\alpha$ on $\mathscr{D}_{L^{2}}^{1}\left(R^{n}\right) \cdot \mathscr{V}\left(\mathbf{R}^{n}\right)$ is a Banach space with the norm

$$
|\alpha|=\sup \left\{\mid\langle\alpha, F>| ; F \in \mathscr{D}_{L^{2}}^{1}(\mathbf{R}),\|F\|_{(1)}=1\right\} .
$$

Then a linear mapping $\widetilde{U}$ from $\mathscr{V}\left(\mathbf{R}^{n}\right)$ into $\mathscr{U}(I)$ is defined by

$$
<\tilde{U} \alpha, \varphi>=\left\langle\alpha, U^{-1} \varphi>\quad\left(\varphi \in H_{0}^{1, B}(I, X)\right)\right.
$$

We have

$$
\left\{\begin{array}{l}
\tilde{U} \mathscr{V}\left(\mathbf{R}^{n}\right)=\mathscr{U}(I) \\
|\alpha|=\|\widetilde{U} \alpha\|
\end{array}\right.
$$

Denote by $q(y)$ a real-valued function on $\mathbf{R}^{n} . q(y)$ is assumed to satisfy the following conditions:

(Q) $q(y)$ is continuously differentiable on $\mathbf{R}^{n}$ and behaves like $O\left(|y|^{-1-\varepsilon}\right)(\varepsilon>0)$ at infinity, i.e., there exist constants $c>0, \rho>0$ such that

17) The Hilbert space $\mathscr{D}_{L^{2}}^{1}\left(\mathbf{R}^{n}\right)$ is defined as the completion of $C_{0}^{\infty}\left(\mathbf{R}^{n}\right)$ in the norm

$$
\|F\|_{11)}^{2}=\int_{\mathbb{R}^{n}}\left\{\sum_{j=1}^{n}\left|\frac{\partial F}{\partial y_{\jmath}}\right|^{2}+|F(y)|^{2}\right\} d x
$$




$$
|q(y)| \leqq c|y|^{-1-\varepsilon} \quad(|y| \geqq \rho)
$$

with $0<\varepsilon<1$.

Let us define $C(r)$ by

$$
\left\{\begin{array}{l}
C(r)=q(r \omega) \times \\
\mathscr{D}(C(r))=D
\end{array}\right.
$$

It is easy to see that $C(r)$ satisfies Assumptions 1.1 and 1.2.

Define a differential operator $H$ by

$$
\left\{\begin{array}{l}
\mathscr{D}(H)=C_{0}^{\infty}\left(\mathbf{R}^{n}\right) \\
H \Phi=-\Delta \Phi+q(y) \Phi .
\end{array}\right.
$$

Then we have

$$
U H \Phi=L U \Phi \quad\left(\Phi \in C_{0}^{\infty}\left(\mathbf{R}^{n}\right)\right)
$$

where

$$
L=-\frac{d^{2}}{d r^{2}}+B(r)+C(r)
$$

Denote by $\mathscr{V}_{1+\varepsilon}\left(\mathbf{R}^{n}\right)$ the set of all $\alpha \in \mathscr{V}\left(\mathbf{R}^{n}\right)$ such that

$$
|\alpha|_{1+\varepsilon}=\sup \left\{\left|<\alpha,(1+r)^{\frac{1+\varepsilon}{2}} F>\right| ; F \in \mathscr{D}_{L^{2}}^{1}\left(\mathbf{R}^{n}\right),\|F\|_{(1)}=1\right\}<\infty .
$$

We have $\tilde{U} \mathscr{V}_{1+\varepsilon}\left(\mathbf{R}^{n}\right)=\mathscr{U}_{1+\varepsilon}(I)$ and $|\alpha|_{1+\varepsilon}=\|\widetilde{U} \alpha\|_{1+\varepsilon}$ for $\alpha \in U_{1+\varepsilon}\left(\mathbf{R}^{n}\right)$.

We now give the definition of the radiative function for $H$ as follows:

Let $k \in \mathbf{C}^{+}$and $\alpha \in \mathscr{V}\left(\mathbf{R}^{n}\right)$. Then $F \in \operatorname{loc} \mathscr{D}_{L^{2}}^{1}\left(\mathbf{R}^{n}\right)^{18)}$ is called the radiative function for $\{H, k, \alpha\}$, if $F$ satisfies the following conditions;

(1) For any $\Phi \in C_{0}^{\infty}\left(\mathbf{R}^{n}\right)$ we have

$$
\left(F,\left(H-\bar{k}^{2}\right) \Phi\right)_{L^{2}\left(\mathbf{R}^{n}\right)}=\langle\alpha, \Phi\rangle .
$$

18) loc $\mathscr{D}_{L^{2}}^{1}\left(\mathbf{R}^{n}\right)$ is the set of all $F(y)$ on $R^{n}$ such that $\psi_{n} F \in \mathscr{D}_{L^{2}}^{1}\left(\mathbf{R}^{n}\right)$ for any $n=1$, $2, \cdots$, where $\psi_{n} \in C_{0}^{\infty}\left(\mathbf{R}^{n}\right), 0 \leqq \psi_{n} \leqq 1$ and

$$
\psi_{n}(x)= \begin{cases}1 & \text { for }|x| \leqq n \\ 0 & \text { for }|x| \geqq n+1 .\end{cases}
$$


(2) The "radiation condition"

$$
\int_{|y| \geqq 1}(1+|y|)^{-1+\varepsilon}\left|-\frac{\partial F}{\partial|y|}-i k F(y)\right|^{2} d y<\infty
$$

holds.

Let $F$ be the radiative function for $\{H, k, 0\}, k \in \mathrm{C}^{+}$. Then, putting $v=U F \in \operatorname{loc} H_{0}^{1, B}(\bar{I}, X)$, we have

$$
\left(\left(v,\left(L-\bar{k}^{2}\right) \varphi\right)\right)_{0}=0 \quad\left(\varphi \in C_{0}^{2, B}(I, X)\right),
$$

and

$$
\left\|v^{\prime}-\frac{n-1}{2 r} v-i k v\right\|_{-1+\varepsilon,(1, \infty)}<\infty .
$$

Modifying slightly the proof of Lemma 2.1, we obtain

$$
\begin{aligned}
\mid v^{\prime}(r) & -\frac{n-1}{2 r} v(r)-\left.i k v(r)\right|^{2} \\
= & \left|v^{\prime}+\left(\operatorname{Im} k-\frac{n-1}{2 r}\right) v(r)\right|^{2}+(\operatorname{Re} k)^{2}|v(r)|^{2} \\
& -2(\operatorname{Re} k) \operatorname{Im}\left(v^{\prime}(r), v(r)\right) \\
= & \left|v^{\prime}(r)+\left(\operatorname{Im} k-\frac{n-1}{2 r}\right) v(r)\right|^{2}+(\operatorname{Re} k)^{2}|v(r)|^{2} \\
& +4(\operatorname{Re} k)^{2}(\operatorname{Im} k)\|v\|_{0,(0, r) .}^{2}
\end{aligned}
$$

If $\operatorname{Im} k \neq 0$, then we see from (5.20)

$$
\begin{aligned}
& \lim _{r_{j} \rightarrow \infty}\|v\|_{0,\left(0, r_{j}\right)}^{2} \\
& \leqq \frac{1}{4(\operatorname{Re} k)^{2}(\operatorname{Im} k)} \lim _{r_{j} \rightarrow \infty}\left|v^{\prime}\left(r_{j}\right)-\frac{n-1}{2 r} v\left(r_{j}\right)-i k v\left(r_{j}\right)\right|^{2}=0
\end{aligned}
$$

along some sequence $\left\{r_{j}\right\}_{j=1}^{\infty}$, and hence $\|v\|_{0}=0$, i.e., $v \equiv 0$. If $\operatorname{Im} k=0$, then we obtain from $(5.20)$

$$
\begin{aligned}
& \left|v^{\prime}(r)-\frac{n-1}{2 r} v(r)-i k v(r)\right|^{2} \\
& \quad \geqq\left|v^{\prime}(r)-\frac{n-1}{2 r} v(r)\right|^{2}+(\operatorname{Re} k)^{2}|v(r)|^{2}
\end{aligned}
$$




$$
\begin{aligned}
& \geqq \frac{1}{2}\left|v^{\prime}(r)\right|^{2}+\left(k^{2}-\frac{(n-1)^{2}}{4 r^{2}}\right)|v(r)|^{2} \\
& \geqq \frac{1}{2}\left\{\left|v^{\prime}(r)\right|^{2}+k^{2}\left|v(r)^{2}\right|\right\}, \quad\left(r \geqq \frac{n-1}{\sqrt{2 k}}\right),
\end{aligned}
$$

whence follows $\lim _{r \rightarrow \infty}\left(\left|v^{\prime}(r)\right|^{2}+k^{2}|v(r)|^{2}\right)=0$. Therefore, proceeding as in the proof of Theorem 2.2, we have $v \equiv 0$. Thus the uniqueness of the radiative function for $H$ has been proved.

Next let $\alpha \in \mathscr{V}_{1+\varepsilon}\left(\mathbf{R}^{n}\right)$ and $k \in \mathbf{C}^{+}$. Since we have $\tilde{U} \alpha \in \mathscr{U}_{1+\varepsilon}(I)$, it follows from Theorem 3.7 that there exists the radiative function $v$ $=v(\cdot, k, \tilde{U} \alpha)$ for $\{L, k, \tilde{U} \alpha, 0\}$. Put

$$
F=U^{-1} v(\cdot, k, \tilde{U} \alpha)
$$

Then $F \in \operatorname{loc} \mathscr{D}_{L^{2}}^{1}\left(\mathbf{R}^{n}\right)$ and it follows from (5.23) that

$$
\left(F,\left(H-\bar{k}^{2}\right) \Phi\right)_{L^{2}\left(\mathbf{R}^{n}\right)}=\left(\left(v,\left(L-\bar{k}^{2}\right) U \Phi\right)\right)_{0}=\langle\widetilde{U} \alpha, U \Phi\rangle=\langle\alpha, \Phi\rangle
$$

holds for any $\Phi \in C_{0}^{\infty}\left(\mathbf{R}^{n}\right)$. Since $v \in H^{-1-\varepsilon}(I, X)$ and $0<\varepsilon<1$, we have $\frac{n-1}{2 r} v \in H^{-1+\varepsilon}((1, \infty), X)$. This together with $v^{\prime}-i k v \in H^{-1+\varepsilon}(I, X)$ implies that $v^{\prime}-i k v-\frac{n-1}{2 r} v \in H^{-1+\varepsilon}((1, \infty), X)$. Hence we obtain

$$
\begin{gathered}
\int_{|y| \geqq 1}(1+|y|)^{-1+\varepsilon}\left|\frac{\partial F}{\partial|y|}-i k F(y)\right|^{2} d y \\
=\left\|v^{\prime}-i k v-\frac{n-1}{2 r} v\right\|_{-1+\varepsilon,(1, \infty)}^{2}<\infty .
\end{gathered}
$$

Therefore it has been shown that $F=U^{-1} v$ is the radiative function for $\{H, k, \alpha\}$. It follows from $v \in H^{-1-\varepsilon}(I, X)$ that $F \in L^{2}\left(\mathbf{R}^{n},(1+|y|)^{-1-\varepsilon} d y\right)$. Thus we obtain

Theorem 5.1. Let $n$ be an integer such that $n \geqq 3$. Let $q(y)$ satisfy the condition (Q). Then for given $k \in \mathbb{C}^{+}$and $\alpha \in \mathscr{V}\left(\mathbb{R}^{n}\right)$ the radiative function $F(\cdot, k, \alpha)$ for $\{H, k, \alpha\}$ is unique. For given $k \in \mathbb{C}^{+}$and $\alpha \in \mathscr{V}_{1+\varepsilon}\left(\mathbb{R}^{n}\right)$ there exists the radiative function $F(\cdot, k, \alpha)$ for $\{H, k, \alpha\}$ such that $F(\cdot, k, \alpha) \in L^{2}\left(\mathbf{R}^{n},(1+|y|)^{-1-\varepsilon} d y\right)$. Denote by $\sigma$ the mapping 


$$
\begin{aligned}
\sigma: & \mathbf{C}^{+} \times v_{1+\varepsilon}\left(\mathbb{R}^{n}\right) \ni(k, \alpha) \\
& \rightarrow F(\cdot, k, \alpha) \in L^{2}\left(R^{n},(1+|y|)^{-1-\varepsilon} d y\right) \cap \operatorname{loc} \mathscr{D}_{L^{2}}^{1}\left(\mathbf{R}^{n}\right) .
\end{aligned}
$$

Then $\sigma$ is continuous as a mapping from $\mathbb{C}^{+} \times \mathscr{V}_{1+\varepsilon}\left(\mathbb{R}^{n}\right)$ into $L^{2}\left(\mathbb{R}^{n},(1+|y|)^{-1-\varepsilon} d y\right)$ and is also continuous as a mapping from $\mathbf{C}^{+}$ 火 $\mathscr{V}_{1+\varepsilon}\left(\mathbf{R}^{n}\right)$ into loc $\mathscr{D}_{L^{2}}^{1}\left(R^{n}\right)$.

\section{References}

[1] Eidus, D. M., The principle of limit amplitude, Uspekhi Mat. Nauk, 24, No. 3 (1969), 91-156. (Russian Mathematical Surveys, 24, No. 3 (1969), 97-169).

[2] Jäger, W., Über das Dirichletsche Aussenraumproblem für die Schwingungsgleichung, Math. Z. 95 (1967), 299-323.

[3] — Zur Theorie der Schwingungsgleichung mit variablen Koeffizienten in Aussengebieten, Math. Z. 102 (1967), 62-88.

[4] — Das asymptotische Verhalten von Lösungen eines Typus von Differentialgleichungen, Math. Z. 112 (1969), 26-36.

[5] — Ein gewöhnlicher Differentialoperator zweiter Ordnung für Funktionen mit Werten in einem Hilbertraum, Math. Z. 113 (1970), 68-98.

[6] Yosida, K., Functional Analysis, Springer, 1965.

[7] Saitō, Y., Spectral and scattering theory for second-order differential operators with operator-valued coefficients, to oppear in Osaka J. Math. 
